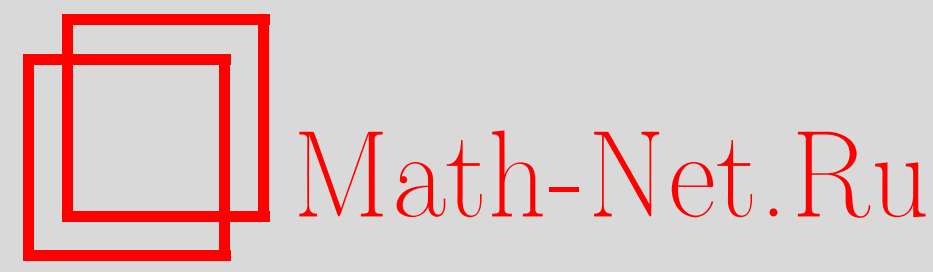

Е. П. Долженко, Е. А. Севастьянов, Аппроксимации со знакочувствительным весом (теоремы существования и единственности), Изв. РАН. Сер. матем., 1998, том 62, выпуск 6, 59-102

DOI: https://doi.org/10.4213/im221

Использование Общероссийского математического портала Math-Net.Ru подразумевает, что вы прочитали и согласны с пользовательским соглашением

http://www . mathnet.ru/rus/agreement

Параметры загрузки:

IP : 54.224 .135 .184

26 апреля 2023 г., 09:12:28 
УДК 517.518 .8

\author{
Е. П. Долженко, Е. А. Севастьянов
}

\title{
Аппроксимации со знакочувствительньм весом (теоремы существования и единственности)
}

\begin{abstract}
Аппроксимации со знакочувствительным весом учитывают, вообще говоря, и модуль ошибки приближения, и ее знак. В работе изучаются вопросы существования, единственности и множественности элемента наилучшего равномерного приближения с заданным знакочувствительным весом $p=\left(p_{-}, p_{+}\right)$посредством функций некоторого семейства $L$ на отрезке $\Delta$ и такие же вопросы об аппроксимациях в линейных нормированных пространствах $\mathscr{L}$ посредством элементов некоторого семейства $L \subset \mathscr{L}$, когда за меру уклонения элемента $x$ от элемента $y$ берется значение $P(x-y)$ некоторого неотрицательного сублинейного функционала $P$. Чрезвычайно важную роль при этом играют жесткость и свобода систем $(p, L)$ и $(P ; L)$. Эти понятия также изучаются в работе, особенно подробно в случае чебышевских подпространств $L$.
\end{abstract}

Библиография: 22 наименования.

\section{Введение}

В отличие от классических весовых аппроксимаций, аппроксимации со знакочувствительным весом учитывают не только модуль разности приближающей функции и приближаемой, но и знак этой разности. Такие аппроксимации содержат в себе, в частности, аппроксимации односторонние, аппроксимации с условием интерполящии в заданных точках и с некоторыми другими условиями. $\mathrm{K}$ таким аппроксимациям сводится также целый ряд экстремальных задач в классах полиномов. Хотя знакочувствительный вес и является непосредственным обобщением веса обычного, большинство результатов рассматриваемой теории и по форме, и по существу необычны. Некоторые из них нам кажутся интересными.

Аппроксимации со знакочувствительным весом являются частным случаем аппроксимаций в линейных пространствах относительно неотрищательного сублинейного функционала, и мы, где это удается, формулируем полученные результаты для общего случая. Накладываемые условия на меру уклонения приближающей функции от приближаемой и на семейства приближающих функций диктуются, как правило, существом дела (например, нигде не предполагается непрерывность веса или его невырожденность). Вводимые здесь понятия жесткости и свободы системы (мера уклонения, семейство приближающих функций), а также понятие $d$-расстояния между весами, как нам кажется, хорошо отражают существо задачи аппроксимации со знакочувствительным весом. Авторы намерены в дальнейшем опубликовать свои результаты об устойчивости задачи знакочувствительной аппроксимации и приложения излагаемой теории к теории чебышевских ужей и хаусдорфовых аппроксимаций. 
Приводимые ниже результаты частично анонсированы авторами в работах 1992-1996 гг. (см. [1]-[10]).

Введем обозначения и дадим определения.

$\mathbf{0 . 0} ;$. Ниже $E$ обозначает некоторое метрическое пространство с метрикой $r$; $\operatorname{diam} L:=\sup \left\{r\left(l_{1}, l_{2}\right): l_{1}, l_{2} \in L\right\}$ - диаметр множества $L \subset E ; M(E)$ - линейное пространство всех (действительнозначных) функций (функционалов) $f$, определенных и ограниченных на $E$ (например, на некотором множестве $E$ числовой прямой $\mathbb{R}$ с обычным расстоянием); $M S(E)$ - множество всех функций, определенных, ограниченных и полунепрерывных сверху на $E ; C(E)$ - линейное пространство ограниченных непрерывных вещественнозначных функций (функционалов) на $E$ $(C(E) \subset M S(E) \subset M(E))$. С нормой $\|f\|_{E}:=\sup \{|f(x)|: x \in E\}$ каждое из множеств $M(E)$ и $C(E)$ представляет собой полное нормированное пространство, $M S(E)$ - полное метрическое пространство с метрикой

$$
\rho(f, g):=\|f-g\|_{E} .
$$

Здесь полунепрерывность понимается в обычном смысле: функционал $f(x)$ (функция), определенный на метрическом пространстве $E$ с метрикой $r$, называется по-

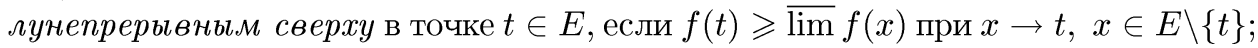
если $f(t) \leqslant \underline{\lim } f(x)$ при $x \rightarrow t, x \in E \backslash\{t\}$, то $f$ полунепрерывен снизу в точке $t$.

Эквивалентное определение: $f$ полунепрерывен сверху (снизу) в точке $t \in E$, если для любого $\varepsilon>0$ существует такое $\delta>0$, что $f(x)<f(t)+\varepsilon$ (соответственно $f(x)>f(t)+\varepsilon)$ при $x \in E, r(x, t)<\delta$. Непрерьвность $f$ в точке $t$ - это полунепрерывность $f$ в $t$ одновременно сверху и снизу.

$\mathbf{0 . 0} ; 2$. Для любой функции $g(x)$, заданной на множестве $E$, ее полунепрерывную сверху регуляризачию (верхнюю функцию Бэра) $\bar{g}$ определим на замыкании $\bar{E}$ множества $E$ равенством

$$
\bar{g}(t):=\varlimsup_{x \rightarrow t} g(x)=\lim _{s \rightarrow 0} \sup \{g(x): x \in E, r(x, t)<s\},
$$

а ее полунепрерывную снизу регуляризацию (нижнюю функцию Бэра) $g$ определим равенством

$$
\underline{g}(t):=\underline{\lim }_{x \rightarrow t} g(x)=\lim _{s \rightarrow 0} \inf \{g(x): x \in E, r(x, t)<s\} .
$$

Очевидно, $g(t)=-\overline{(-g(t))}$, на множествах $\bar{E}$ и $E$ функция $\bar{g}$ полунепрерывна сверху, а $g$ полунепрерьвна снизу, $\bar{g}(x)=g(x)$ на $E$ при полунепрерывности сверху $g(x)$ на $E$ и $\underline{g}(x)=g(x)$ на $E$ при полунепрерывности снизу $g(x)$ на $E$. Отметим еще, что существуют такие ограниченные функции $g(x)$ на $[0,1]$, что $\underline{g}(x)<g(x)<\bar{g}(x) \quad \forall x$.

0.0; 3. Упорядоченную пару $p=\left(p_{-}, p_{+}\right)$неотрицательных функций $p_{-}(x)$ и $p_{+}(x)$, определенных на некотором множестве $E$ и принимающих, вообще говоря, также и значение $+\infty$, назовем знакочувствительным весом на $E$; такой вес $p$ назовем ограниченным, полунепрерывнылм сверху, если таковы $p_{-}$и $p_{+} ;$при $p(x)=(0,0)$ вес $p$ назовем вырожсенным м в точке $x$, при $p_{-}(x)>0$ и $p_{+}(x)>0-$ 
невырожсденным лувырожсденным; при $p(x) \equiv(0,0)$ назовем $p$ тривиальным, при $p(x) \not \equiv(0,0)-$ нетривиальным.

Всюду ниже термин “вес" означает знакочувствительный вес. Положим

$$
\|p\|=\|p\|_{E}:=\max \left\{\left\|p_{-}\right\|_{E},\left\|p_{+}\right\|_{E}\right\}
$$

При $\|p\|=1$ вес $p$ назовем нормированным. Полунепрерывной сверху регуляризаиией веса $p=\left(p_{-}, p_{+}\right)$назовем вес $\bar{p}=\left(\bar{p}_{-}, \bar{p}_{+}\right)$.

$\mathbf{0 . 0} ; 4$. Ниже $a^{+}=\max \{a, 0\}, a^{-}=(-a)^{+}\left(a=a^{-}-a^{+},|a|=a^{-}+a^{+}\right)$. Если функция $f$ определена на $E$ и принимает, вообше говоря, и значения $+\infty$, и $-\infty$, а $p(x)$ - вес на $E$, то положим

$$
(f, p)(x):=f^{+}(x) p_{+}(x)-f^{-}(x) p_{-}(x), \quad|f|_{p, E}:=\|(f, p)\|_{E} \leqslant\|p\|_{E} \cdot\|f\|_{E}
$$

$($ считаем $0 \cdot( \pm \infty)=( \pm \infty) \cdot 0=0, a \cdot( \pm \infty)=( \pm \infty) \cdot a= \pm \infty$ при $0<a \leqslant+\infty)$.

$\mathbf{0 . 0 ;} 5$. Очевидно, $|f|_{p, E}$ - положительный сублинейный функционал на $M(E)$, ограниченный и непрерывный при $\|p\|_{E}<\infty$ (вообще говоря, $|-f|_{p, E} \neq|f|_{p, E}$ ). Напомним, что неотрицательный функционал $P(f)$ на некотором линейном нормированном пространстве $\mathscr{L}$ (вообще говоря, принимающий и значение $+\infty$ ) называется положительным сублинейным, если он: а) выпукльй; б) положительно однородный (это эквивалентно тому, что

$$
0 \leqslant P(f+g) \leqslant P(f)+P(g) \leqslant+\infty, \quad P(k f)=k P(f)
$$

для любых $f, g \in \mathscr{L}$ и $k$ - const $>0)$.

Положительный сублинейный функционал $P(f)$ называют строго выnукльлм, если $P(f+g)<P(f)+P(g)$ для любой пары неколлинеарных элементов $f, g \in \mathscr{L}$.

Отметим, что положительные сублинейные функционалы с тривиальным ядром $N(P):=\{f: f \in \mathscr{L}, P(f)=0\}$, т.е. состояшим лишь из элемента $f=0$, в качестве "масштабной функции" в конечномерном пространстве рассматривал Г. Минковский [11]. Такие же функционалы в качестве несимметричных норм рассматривались М. Г. Крейном и А. А. Нудельманом в [12], причем там под именем "( $\left.\varphi_{+}, \varphi_{-}\right)$-норм" также использовались и $p$-нормы для случая, когда $E=[a, b]$, а $p_{-}=1 / \varphi_{-}, p_{+}=1 / \varphi_{+}$, где функции $\varphi_{-}$и $\varphi_{+}$непрерьвны и строго положительны на отрезке $[a, b]$.

$\mathbf{0 . 0 ;} 6$. Если $P$ - произвольный функционал на некотором линейном нормированном пространстве $\mathscr{L}$ с нормой $\|\cdot\|, L \subset \mathscr{L}$, то полагаем

$$
\|P\|_{L}:=\sup \left\{\frac{|P(l)|}{\|l\|}: l \in L, l \neq 0\right\}, \quad\|P\|:=\|P\|_{\mathscr{L}}
$$

(при $\|P\|<\infty$ функционал $P$ называется ограниченны.м).

0.0; 7. Очевидно,

$$
\left\|\left.\left|\cdot{ }_{p, E}\left\|_{C(E)}=\right\|\right| \cdot\right|_{p, E}\right\|_{M(E)}=\|p\|_{E} .
$$


$\mathbf{0 . 0 ; ~ 8 . ~ Е с л и ~} P$ - выпуклый неотрищательный функционал, то

$$
\begin{gathered}
|P(f)-P(g)| \leqslant \max \{P(f-g), P(g-f)\} \leqslant\|P\| \cdot\|f-g\| \\
(P(f) \leqslant P(g)+P(f-g) \leqslant P(g)+\|P\| \cdot\|f-g\|, \\
P(g) \leqslant P(f)+P(g-f) \leqslant P(f)+\|P\| \cdot\|g-f\|) .
\end{gathered}
$$

Легко доказывается следующее свойство функционала $|\cdot|_{p, E}$.

0.1. ТЕОремА. Пусть $p=\left(p_{-}, p_{+}\right)$- не обязательно конечный вес на некотором множестве $E$. Тогда сублинейный функиионал $P(f)=|f|_{p, E}$ на $M(E)$ полунепрерывен снизу, а при ограниченном р ограничен и непрерывен.

Действительно, при $f \in M(E)$ по заданному $\varepsilon>0$ найдем такое $t \in E$, что $|(f, p)(t)|>|f|_{p, E}-\varepsilon$ в случае $|f|_{p, E}<\infty$ или $|(f, p)(t)|>\varepsilon$ в случае $|f|_{p, E}=\infty$. Тогда если $f_{n} \in M(E),\left\|f_{n}-f\right\|_{E} \rightarrow 0(n \rightarrow \infty)$, то при $|f|_{p, E}<\infty$

$$
\underline{\lim }\left|f_{n}\right|_{p, E} \geqslant \underline{\lim }\left|\left(f_{n}, p\right)(t)\right|>|f|_{p, E}-\varepsilon
$$

или при $|f|_{p, E}=\infty$

$$
\underline{\lim }\left|f_{n}\right|_{p, E}>\varepsilon
$$

В любом случае отсюда получаем

$$
\underline{\lim }\left|f_{n}\right|_{p, E} \geqslant|f|_{p, E},
$$

т.е. функционал $|\cdot|_{p, E}$ полунепрерывен снизу. Его ограниченность и непрерывность в случае ограниченности веса $p$ следует из определения $|\cdot|_{p, E}$ (cм. п. 0.0;4)

0.2. Уклонением $l \in \mathscr{L}$ от $f \in \mathscr{L}$ относительно сублинейного функционала $P$ назовем величину $P(l-f)$ (вообше говоря, $P(l-f) \neq P(f-l)$ ); наименьиим уклонением непустого мнохсества $L \subset \mathscr{L}$ от әлемента $f \in \mathscr{L}$ относительно функционала $P$ называется величина

$$
E(P ; L, f):=\inf \{P(l-f): l \in L\}
$$

$l(P ; L, f)$ обозначает соответствующий элемент наилучшего приближения (он может не существовать или быть неединственным):

$$
P(l(P ; L, f)-f)=E(P ; L, f)
$$

$E(L, f)$ - наименьшее уклонение множества $L \subset \mathscr{L}$ от элемента $f \in \mathscr{L}$ по норме $\|\cdot\|$ пространства $\mathscr{L}$. При $f \in \mathscr{L}$ и непустом $L \subset \mathscr{L}$ через $A(P ; L, f)$ обозначим множество всех элементов $l(P ; L, f)$ наилучшего приближения для $f(A(P ; L, f)$ может быть пустым). При $P(\cdot)=|\cdot|_{p, E}$ пишем $E(p, L, f), l(p, L, f)$ и $A(p, L, f)$ вместо $E(P ; L, f), l(P ; L, f)$ и $A(P ; L, f)$ соответственно.

Отметим, что если $E(p, L, f)<\infty$ и $p_{-}(x)=p_{+}(x)=\infty$ на некотором множестве $F \subset E$, то $l(p, L, f ; x)$ совпадает с $f(x)$ на $F$, т.е. в этом случае приближение сопровождается интерполяцией на $F$ приближаемой функции. Если же 
$p_{-}(x)=p_{+}(x)=0$ на некотором множестве $G \subset E$, то имеем другой крайний случай: на величину $E(p, L, f)$ никак не влияют значения функций $f, l \in L$ на $G$.

Заметим еще, что в задачах аппроксимации непрерывных функций непрерывными же (например, полиномами) достаточно ограничиться лишь полунепрерывными сверху весами. Действительно, если $\bar{p}=\left(\bar{p}_{-}, \bar{p}_{+}\right)-$полунепрерывная сверху регуляризация веса $p=\left(p_{-}, p_{+}\right)$, то, как легко видеть, при $r=\bar{p}$ для любой пары функций $l, f \in C(E)$ имеем

$$
|l-f|_{r, E}=|l-f|_{p, E},
$$

и если при этом $L$ состоит лишь из таких функций $l$, то $E(\bar{p}, L, f)=E(p, L, f)$. Если $f$ и все $l \in L$ равномерно непрерывны на $E$, то $E(p, L, f)=E\left(\bar{p}, L^{*}, f^{*}\right)$, где $f^{*}$ - непрерьвное продолжение $f$ на $\bar{E}$, а семейство $L^{*} \operatorname{cocтоит~из~функций~} l \in L$, по непрерывности продолженных на $\bar{E}$. Итак, если множество $E$ замкнуто, то при аппроксимации функций $f \in C(E)$ функциями некоторого семейства $L \subset C(E)$ можно ограничиться лишь весами $p$, полунепрерьвными сверху на $E$.

\section{Глава 1. Пространство знакочувствительных весов}

В гл. 3 знакочувствительный вес будет использован непосредственно для полиномиальных и более обших аппроксимаций. Здесь же мы рассмотрим само пространство знакочувствительных весов со специальной метрикой $d(p, q)($ cм. [1]-[5]).

Очевидно, с метрикой

$$
r(p, q)=\max \left\{\left\|p_{-}-q_{-}\right\|_{E},\left\|p_{+}-q_{+}\right\|_{E}\right\}
$$

множество всех ограниченных знакочувствительных весов превращается в (несепарабельное) полное метрическое пространство. Однако такая характеристика близости весов плохо отражает специфику задачи наилучшей равномерной аппроксимации функций с, вообще говоря, разрывным весом. Вводимая ниже метрика $d(p, q)$, по-видимому, хорошо приспособлена к этой задаче. С метрикой $d(p, q)$ множество всех ограниченных полунепрерывных сверху знакочувствительных весов на отрезке $\Delta=[a, b]$ представляет собой полное сепарабельное метрическое пространство. Вьше отмечалось, что именно полунепрерывными сверху весами достаточно ограничиться в случае приближения непрерывных функций непрерывными же функциями. В пространстве всех ограниченных знакочувствительных весов величина $d(p, q)$ уже не является метрикой в собственном смысле этого слова, поскольку может быть $d(p, q)=0$ при не равных между собой знакочувствительных весах $p$ и $q$ (например, так будет при $p=(k, 1-k), q=(1-k, k)$, где $k(x)=0$ при иррациональных $x \in[0,1], k(x)=1$ при рациональных $x \in[0,1])$. Однако и в этом случае величина $d(p, q)$ хорошо связана с задачей весовой аппроксимации и некоторыми экстремальными задачами.

1.1. Перед определением $d$-расстояния $d(p, q)$ напомним некоторые необщеизвестные понятия.

1) Расстояние Минковского $\rho_{M}$ на плоскости $x O y$ между точками $(x, y)$ и $(u, v)$ определяется равенством

$$
\rho_{M}((x, y),(u, v))=\max \{|x-u|,|y-v|\}
$$


2) Множество $F$ на плоскости $x O y$ называется выпуклым относительно оси $O y$, если вместе с любыми двумя точками из $F$, имеющими одну и ту же абсциссу, в $F$ входит соединяющий их вертикальный отрезок.

3) Дополненны.м графиком $F(f)$ функции $y=f(x)$ (вообще говоря, неоднозначной) называется наименьшее замкнутое множество, выпуклое относительно оси $O y$ и содержащее график этой функции. Дополненный график ограниченной функции, заданной на промежутке (т.е. на замкнутом или полузамкнутом отрезке, на открытом интервале), является замкнутым связным множеством (континуумом, если промежуток конечен).

4) Хаусдорфовым расстоянием $h(A, B)$ между двумя ограниченными множествами $A$ и $B$ на плоскости $x O y$ называется инфимум всех таких $r>0$, что $A$ и $B$ содержатся друг у друга в $r$-окрестностях Минковского. Хаусдорфовыл $р$ расстоянием между двумя ограниченными функииями $g$ и $h$, определенными на некотором множестве $E \subset \mathbb{R}$, называется величина $H(g, h):=h(F(g), F(h))$ - хаусдорфово расстояние между их дополненными графиками (см. [13]).

1.2. ОПРЕДЕЛЕНИЕ. Для двух ограниченных функций, $g(x)$ и $h(x)$, определенных на некотором множестве $E \subset \mathbb{R}$, через $d(g, h)$ обозначим инфимум всех чисел $r>0$, при которых график максимальной для $g$ и $h$ функции

$$
M(g, h ; x):=\max \{g(x), h(x)\}
$$

содержится в $r$-окрестности Минковского каждого из дополненных графиков $F(g)$ и $F(h)$ этих функций.

Для ограниченных на множестве $E$ знакочувствительных весов $p=\left(p_{-}, p_{+}\right)$и $q=\left(q_{-}, q_{+}\right)$положим

$$
d(p, q):=\max \left\{d\left(p_{-}, q_{-}\right), d\left(p_{+}, q_{+}\right)\right\} .
$$

Величины $d(g, h)$ и $d(p, q)$ назовем $d$-расстояниям между функциями $g, h$ и весами $p, q$ соответственно.

1.3. Легко доказываются следующие свойства $d$-расстояния:

$$
\text { 1.3; } 1 . \quad d(g, \bar{g})=0 \quad \forall g \in M(E) \quad(E \subset \mathbb{R}) .
$$

1.3; 2 . При $f, g \in M(E)$ для $d$-расстояния, хаусдорфова $H(f, g)$ и равномерного $\|f-g\|_{E}$ расстояний имеем неравенства

$$
d(f, g) \leqslant H(f, g) \leqslant\|f-g\|_{E}
$$

и если $E=\Delta=\langle a, b\rangle-$ промежуток, то также и равенство

$$
H(f, g)=\max \{d(f, g), d(-f,-g)\}
$$

в случае неотрицательных $f, g \in M(E)$ и знакочувствительных ограниченных весов $p, q$ имеем также неравенства

$$
\left|\|f\|_{E}-\|g\|_{E}\right| \leqslant d(f, g), \quad\left|\|p\|_{E}-\|q\|_{E}\right| \leqslant d(p, q) .
$$


1.3; 3 . Если $\chi_{A}(x)$ и $\chi_{B}(x)$ - характеристические функции множеств $A$ и $B$, то $d\left(\chi_{A}, \chi_{B}\right) \leqslant h(A, B)$ (см. 1.1, п. 4)), причем $d\left(\chi_{A}, \chi_{B}\right)=h(A, B)$ при $h(A, B) \leqslant 1$ и $d\left(\chi_{A}, \chi_{B}\right)=1$ при $h(A, B) \geqslant 1$.

1.3; 4 . Величина $d(f, g)$ может быть малой тогда, когда велики равномерное, интегральное или хаусдорфово расстояния между $f$ и $g$.

1.4. Семейство всех ограниченных и полунепрерывных сверху весов $p=$ $\left(p_{-}, p_{+}\right)$на множестве $E \subset \mathbb{R}$ с метрикой $d(p, q)$ обозначим $\operatorname{DS}(E)$.

1.5. ТеоремА. Пусть $\Delta$ - некоторый промежуток на $\mathbb{R}$. Тогда $\operatorname{DS}(\Delta)$ является полным метрическим пространством, сепарабельным при конечном $\Delta$ и несепарабельным в противном случае.

ДокАЗАТЕЛЬСтво. Достаточно проверить, во-первых, что пространство $\operatorname{DS}^{1}(\Delta)$ всех неотрицательных ограниченных и полунепрерывных сверху функций на $\Delta$ с $d$-метрикой является полным метрическим пространством и, во-вторых, что это пространство сепарабельно в случае конечного промежутка $\Delta$ и несепарабельно в противном случае. Первая часть этого утверждения доказана в [5]. Там же доказана сепарабельность $\operatorname{DS}^{1}(\Delta)$ в случае конечного $\Delta$. В случае бесконечного $\Delta$ (пусть, например, $\Delta=[0,+\infty)$ ) обозначим через $f(x)$ функцию, равную единище при $x \in[0,1]$, нулю при $x \in(1,2)$ и продолженную вправо периодически с периодом 2. Тогда для любых функций $g, h$ несчетного семейства $A=\left\{f_{t}(x):=f(x /(1+t)): 0<t<1 / 8\right\}$ имеем $d(g, h)>1 / 8$, так что пространство $\operatorname{DS}^{1}(\Delta)$ несепарабельно.

Действительно, пусть $g=f_{t}, h=f_{s}, 0<t<s<1 / 8$, и натуральное $n$ выбрано из условия

$$
\frac{1}{4(2 n+1)}<s-t<\frac{1}{2(2 n+1)} .
$$

На интервале $(a=(1+t)(2 n+1),(1+s)(2 n+1)=a+(s-t)(2 n+1))$ имеем тогда $g(x)=0, h(x)=1$. Так как длина этого интервала больше $1 / 4$, то $d(g, h)>1 / 8$, и теорема доказана.

1.6. Отметим, что если $\Delta$ - промежуток на $\mathbb{R}$, то для любой тройки функций $g, h, r$ из $M(\Delta)$ имеем соотношения (см. [5]):

1) $d(g, h) \geqslant 0 ; 2) d(g, h)=d(h, g) ; 3) d(g, h) \leqslant d(g, r)+d(r, h)$.

1.7. Естественно возникает вопрос о связи сходимости по метрике $d$ с другими видами сходимости. Легко построить последовательность непрерывных функций, сходящуюся к непрерывной же функции по метрике $d$, но расходящуюся в каждой точке $x$. Для этого возьмем функцию $g(x)$, линейно возрастаюшую от 0 до 1 на отрезке [0,1/6], равную 1 на $[1 / 6,1 / 2]$, линейно убывающую от 1 до 0 на $[1 / 2,2 / 3]$ и равную 0 на $[2 / 3,1]$, продолженную периодически с периодом 1 на всю ось $O x$. Положим

$$
g_{k}(x)=g\left(x-\frac{k}{9}\right), \quad k=0,1, \ldots, 8,
$$

и построим последовательность функций $\left\{f_{n}(x)\right\}, n=1,2, \ldots$, занумеровав подряд функции $\left\{g_{k}(x)\right\}, k=0, \ldots, 8$, затем $\left\{g_{k}(2 x)\right\}, k=0, \ldots, 8$, затем $\left\{g_{k}(3 x)\right\}$, 
$k=0, \ldots, 8$, и т.д. Очевидно, $d\left(f_{n}, 1\right) \rightarrow 0$, и последовательность $\left\{f_{n}(x)\right\}$ расходится всюду. Как нетрудно видеть, эта последовательность не сходится также и ни в какой интегральной метрике, и даже по мере ни на каком отрезке. В хаусдорфовой метрике последовательность $\left\{f_{n}(x)\right\}$ сходится, но предельной для нее является многозначная функция $f$, множество значений которой в каждой точке $x$ есть отрезок $[0,1]$.

1.8. Теорема. Пусть $Е$ - компакт на $\mathbb{R}$. Для того чтобы множество $A \subset \operatorname{DS}(E)$ было компактом, необходимо и достаточно, чтобы оно было замкнутым и ограниченным.

ДоказАТельСтво. Необходимость очевидна. Достаточность следует из соответствующей теоремы хаусдорфовой метрики $H(f, g)$ (см. [21, §21, VII, теорема 2]) и неравенства $d(f, g) \leqslant H(f, g)$ из п. $1.3 ; 2$.

\section{Глава 2. Жесткость и свобода системы $\S 1$. Определения и простейшие свойства}

Сушественную роль в рассматриваемых ниже вопросах играют следуюшие числовые характеристики системы $(P ; L)$, состоящей из меры уклонения $P(\cdot)$ (например, $P(\cdot)$ - сублинейный функционал на некотором линейном нормированном пространстве $\mathscr{L}$, в частности, $P(\cdot)=|\cdot|_{E, p}$, где $p=\left(p_{-}, p_{+}\right)$- знакочувствительный вес на $E \subset \mathbb{R}$, а $\mathscr{L}=M(E))$ и некоторого множества $L \subset \mathscr{L}$, посредством элементов которого происходит приближение элемента $f \in \mathscr{L}$, когда за величину уклонения $l \in L$ от $f$ берется $P(l-f)$.

2.0. В случае произвольного неотрицательного функционала $P$, определенного на линейном нормированном пространстве $\mathscr{L}$ с нормой $\|\cdot\|$, содержащем некоторое множество $L \neq\{0\}$, величины

$$
\begin{aligned}
S(P ; L) & :=\inf \left\{\frac{P(l)}{\|l\|}: l \in L, l \neq 0\right\} \\
W(P ; L) & :=\sup \left\{\frac{\|l\|}{P(l)}: l \in L, l \neq 0\right\}
\end{aligned}
$$

назовем соответственно жесткостью и свободой системы $(P ; L)$ (как обычно, считаем $a / 0=\infty$ при $a>0, a / \infty=0$ при $|a|<\infty)$. Если на некотором множестве $E$ заданы знакочувствительный вес $p=\left(p_{-}, p_{+}\right)$и некоторое семейство $L$ ограниченных функций $l(x)$, не сводящееся к единственной функции $l(x) \equiv 0$, то величины

$$
\begin{aligned}
S(p, L) & :=\inf \left\{\frac{|l|_{p, E}}{\|l\|_{E}}: l \in L, l \not \equiv 0\right\}, \\
W(p, L) & :=\sup \left\{\frac{\|l\|_{E}}{|l|_{p, E}}: l \in L, l \neq 0\right\}, \quad L \subset M(E),
\end{aligned}
$$

назовем соответственно жесткостью и свободой системы $(p, L)$ на множестве $E$ $\left(S(p, L)=S\left(|\cdot|_{p, E} ; L\right), W(p, L)=W\left(|\cdot|_{p, E} ; L\right)\right)$. Если при этом функции семейства $L$ заданы также на некотором множестве $F \subset \mathbb{R}$ и ограничены на нем, то 
мы можем определить величины $S(p, L, F)$ и $W(p, L, F)$, заменяя в $(2.0 ; 3)$ и $(2.0 ; 4)$ $\|l\|_{E}$ на $\|l\|_{F}$. При этом уже нет нужды требовать ограниченности функций из $L$ на множестве $E$. Очевидно, $S(p, L, E)=S(p, L), W(p, L, E)=W(p, L)$.

Отметим некоторые простейшие свойства жесткости и свободы:

1) $W(P ; L)=1 / S(P ; L), S(P ; L)=1 / W(P ; L)$;

2) $0 \leqslant S(P ; L) \leqslant\|P\|_{L}, \infty \geqslant W(P ; L) \geqslant 1 /\|P\|_{L}($ cM. $(0.0 ; 6))$,

$0 \leqslant S(p, L) \leqslant\|p\|, \infty \geqslant W(p, L) \geqslant 1 /\|p\|($ см. $(0.0 ; 7))$

3) если $L_{1} \subset L$, то $W\left(P ; L_{1}\right) \leqslant W(P ; L)$;

4) если $P$ - сублинейный функционал на $\mathscr{L}, L \subset \mathscr{L}, t$ - const $>0$, то

$$
S(t P ; L)=t S(P ; L), \quad W(t P ; L)=\frac{1}{t} W(P ; L),
$$

и если $p=\left(p_{-}, p_{+}\right), t p:=\left(t p_{-}, t p_{+}\right)$, то

$$
S(t p, L)=t S(p, L), \quad W(t p, L)=\frac{1}{t} W(p, L)
$$

5) $W(P ; L)=S(P ; L)=W(p, L)=S(p, L)=1$ при $P(\cdot)=\|\cdot\|, p(x) \equiv(1,1)$;

$6)$ если $p=\left(p_{-}, p_{+}\right), q=\left(q_{-}, q_{+}\right), p_{-}(x) \leqslant q_{-}(x)$ и $p_{+}(x) \leqslant q_{+}(x)$ при всех $x \in E$, то $S(p, L) \leqslant S(q, L)$.

Рассмотрим еще одно обобшение понятий жесткости и свободы: пусть $L$ - некоторое множество в линейном нормированном пространстве $\mathscr{L}, \mathbb{P}$ - семейство неотрицательных сублинейных функционалов на $\mathscr{L}$. Жесткостью и свободой суперcистемы $(\mathbb{P} ; L)$ назовем соответственно величины

$$
\begin{gathered}
S(\mathbb{P} ; L):=\inf \left\{\frac{P(l)}{\|l\|}: l \in \mathscr{L}, l \neq 0, P \in \mathbb{P}\right\}, \\
W(\mathbb{P} ; L):=\sup \left\{\frac{\|l\|}{P(l)}: l \in \mathscr{L}, l \neq 0, P \in \mathbb{P}\right\} .
\end{gathered}
$$

Если р - некоторое семейство знакочувствительных весов на множестве $E$, $L \subset M(E)$, то жесткостью и свободой суперсистемы $(\mathbf{p}, L)$ назовем соответственно $S(\mathbb{P} ; L)$ и $W(\mathbb{P} ; L)$ с $\mathbb{P}=\left\{|\cdot|_{p, E}: p \in \mathbf{p}\right\}$.

Дальнейшие обобщения введенных понятий мы получим, если на пространстве $\mathscr{L}$ вместо нормы $\|\cdot\|$ возьмем некоторьй функционал $Q$ или некоторое семейство функционалов $\mathbb{Q}$. Например, пусть конечные функционалы $P$ и $Q$ определены на линейном пространстве $\mathscr{L}, L \subset \mathscr{L}, Q(l) \not \equiv 0$ на $L$. Жесткостью и свободой системы $(P, Q ; L)$ назовем соответственно величины

$$
S(P / / Q ; L):=\inf \left\{\left|\frac{P(l)}{Q(l)}\right|\right\}, \quad W(Q / / P ; L):=\sup \left\{\left|\frac{Q(l)}{P(l)}\right|\right\}
$$

где inf и $\sup$ берутся по всем $l \in L$ при условии $|P(l)|+|Q(l)|>0$. Очевидно,

$$
\begin{gathered}
W(P / / Q ; L)=\frac{1}{S}(Q / / P ; L), \quad S(P / / Q ; L) \leqslant \frac{S(P ; L)}{S(Q ; L)}, \\
\|P\|_{L}=W(|P| / /\|\cdot\| ; L) .
\end{gathered}
$$


Нетрудно видеть, что при замене $\|\cdot\|$ на конечный сублинейный функционал $Q(\cdot)$ остаются в силе некоторые теоремы этой и следующих глав, например теорема 3.20 , где вместо $\operatorname{diam} A(\cdot)$ теперь должен быть $\operatorname{diam}_{Q} A(\cdot):=\sup \{Q(l-m)$ : $l, m \in A(\cdot)\}$.

Следующие два простых утверждения непосредственно вытекают из определений.

2.1. ТЕОРемА. Пусть $P$ - произвольный полунепрерывный снизу сублинейный функционал на линейном нормированном пространстве $\mathscr{L}, L \subset \mathscr{L}$, множество $L_{0}=\{l /\|l\|: l \in L, l \neq 0\}$ является компактом (это так, если, например, $L$ - конечномерное подпространство в $\mathscr{L})$. Тогда для бесконечности свободь $W(P ; L)$ необходимо и достаточно, чтобь для некоторого $l \in L, \quad l \neq 0$, билло $P(l)=0$.

2.2. ТЕОРЕМА. Пусть $P$ - неотрицательный функционал на линейном нормированном пространстве $\mathscr{L}, L \subset \mathscr{L}, W(P ; L)<\infty$. Тогда для любого әлемента $l \in L$ имеем

$$
\|P\|_{L}^{-1} P(l) \leqslant\|l\| \leqslant W(P ; L) P(l)=(S(P ; L))^{-1} P(l) .
$$

Если $P$ и L удовлетворяют условиям теоремы 2.1, то равенство в правом неравенстве достигается при некотором $l \in L$.

Ниже мы укажем условия конечности свободы $W(p, L)$ в случае чебышевского подпространства $L$ (теорема 2.7 ) и более общих функциональных семейств (теорема 2.12).

\section{§ 2. Случай чебышевского подпространства}

Если в некоторой окрестности точки $t \in \mathbb{R}$ функция $f(x)$ непрерьвна, в точке $t$ равна нулю и отлична от нуля в остальных точках этой окрестности, то имеет место один из двух случаев:

1 ) при переходе $x$ через $t$ функция $f(x)$ меняет знак;

2) при этом переходе $f(x)$ не меняет знака.

В первом случае $t$ называем нечетным нулем функции $f(x)$ и полагаем $n(t)=$ $n(f, t)=1$, во втором - четным нулем ее и полагаем $n(t)=n(f, t)=2$. Число $n(t)$ назовем четностью нуля функции $f(x)$ в точке $t$. Если $t$ - концевая точка промежутка $\Delta$, на котором $f(x)$ задана и непрерывна, $f(t)=0$, а $f(x) \neq 0$ вблизи $t$ при $x \neq t$, то считаем $t$ нечетным нулем и полагаем $n(t)=1$. Если на промежутке $\Delta$ непрерывная функция $f(x)$ имеет конечное число нулей, то сумму $n(f, \Delta)$ их четностей назовем суммарной четностью нулей функции $f$ на промежутке $\Delta$. Если $f$ не имеет нулей на $\Delta$, полагаем $n(f, \Delta)=0$.

2.3. ОПРЕДЕЛЕНИЕ. Конечномерное подпространство $L$ пространства функций, непрерывных (не обязательно ограниченных) на некотором промежутке $\Delta \subset$ $\mathbb{R}$, называется чебышевским подпространством на $\Delta$, если каждая функция $l \in L$, не тождественная нулю, имеет на $\Delta$ не более $n-1$ нулей, где $n=\operatorname{dim} L-$ линейная размерность $L$ (или, что то же, если $n(l, \Delta) \leqslant n-1$ для каждой не тождественной нулю функции $l \in L$; см., например, [14, с. 23]). 
Линейно независимую систему функций $\left\{\varphi_{k}(x)\right\}_{k=1}^{n}, n \geqslant 1$, определенных и непрерывных на некотором промежутке $\Delta$, называют чебышевской (на $\Delta$ ), если чебьшевским (на $\Delta$ ) является линейное подпространство, порожденное этой системой (т.е. пространство всех полиномов $l=c_{1} \varphi_{1}+c_{2} \varphi_{2}+\cdots+c_{n} \varphi_{n}$ с действительными коэффициентами $c_{k}$ ).

Примеры чебышевских подпространств $L$ дают семейства полиномов: алгебраических степени $\leqslant n-1$ на любом промежутке $\Delta, n \geqslant 1, \operatorname{dim} L=n$, а также тригонометрических порядка $\leqslant n-1$ на промежутке $\Delta=[0,2 \pi), n \geqslant 1, \operatorname{dim} L=2 n-1$.

Следующая лемма нужна для доказательства теорем 2.5 и 3.5.

2.4. ЛЕмма (о нулях элементов чебьшевского подпространства). Пусть $L-$ чебышевское подпространство на промежутке $\Delta=\langle a, b\rangle \quad(-\infty \leqslant a<$ $b \leqslant+\infty), \quad X=\left\{x_{1}, x_{2}, \ldots, x_{j}\right\}$-некоторое множество из $j \leqslant n-1$ различных точек на $\Delta$ с предписанными четностями $n\left(x_{k}\right)$ соответственно, равными 1 или 2, причем если $x_{k}$ - концевая точка промежутка $\Delta$, то $n\left(x_{k}\right)=1$. Пусть $m:=n\left(x_{1}\right)+\cdots+n\left(x_{j}\right) \leqslant n-1$. Тогда существует функиия $l \in L$, для которой каждая точка $x_{k}$ является нулем четности $n\left(x_{k}\right)$ соответственно. При этом $l(x) \neq 0$ при $x \in \Delta \backslash X$ за одним возможным исключением: именно, когда разность $n-m$ четна и ровно одна концевая точка промежутка $\Delta$ принадлежит разности $\Delta \backslash X$. В этом исключительном случае $l(x)$ может иметь нечетный нуль в упомянутой концевой точке.

ДокАЗАТЕЛЬСтво существования нужной функции $l$ в случае, когда разность $n-m$ нечетна, дано в $[14$, c. 24,25$]$. Если же эта разность четна и один из конщов промежутка $\Delta=\langle a, b\rangle$, скажем точка $a$, принадлежит $\Delta \backslash X$, то, присоединив $a$ $\mathrm{K} X$, по предыдущему найдем функцию $l(a ; x)$, равную нулю на $X$ и в точке $a$ и отличную от нуля при остальных $x \in \Delta$. Если же и $a$ и $b$ принадлежат $\Delta \backslash X$ (т.е. если $\Delta=[a, b], X \subset(a, b))$, то для только что построенной функции $l(a ; x)$ имеем $l(a ; b) \neq 0$. Строим аналогично еше одну функцию (обозначим eе $l(b ; x)$ ), имеющую на $(a, b)$ нули в тех же точках и той же четности, что и $l(a ; x), l(b ; x) \neq 0$ на $(a, b) \backslash X$, но при этом $l(b ; b)=0, l(b ; a) \neq 0$. Домножив в случае необходимости $l(b ; x)$ на $(-1)$, добьемся при всех $x \in(a, b) \backslash X$ выполнения условия

$$
\operatorname{sign} l(b ; x)=\operatorname{sign} l(a ; x) .
$$

Тогда функция $l(x):=l(a ; x)+l(b ; x)$ имеет в точках множества $X$ нули тех же четностей, что $l(a ; x)$ и $l(b ; x)$, а на $\Delta \backslash X$ она отлична от нуля. Лемма доказана.

Приводимая ниже теорема 2.7 дает необходимое и достаточное условия на знакочувствительный вес $p$ для конечности свободы системы $(p, L)$ в случае промежутка $E=\langle a, b\rangle$ и чебышевского подпространства $L$ на $\langle a, b\rangle$. Интересно, что в эти условия входит лишь линейная размерность $L$.

2.5. ТЕОРемА. Пусть $L-n$-мерное чебишевское подпространство на промежутке $\Delta=\langle a, b\rangle \quad(-\infty \leqslant a<b \leqslant+\infty), \quad p=\left(p_{-}, p_{+}\right)-$произвольний знакочувствительный вес на $\Delta$ (не обязательно конечный), $F$ - некоторое подмножество этого промежутка, состоящее не менее чем из $n$ точек.

Тогда если $W(p, L, F)<\infty$ (т.е. $S(p, L, F)>0)$, то выполняется одно и только одно из следующих двух условий: 
а) на $\Delta$ имеется возрастающая последовательность из $n+1$ точек, в которых функции $p_{-}(x)$ и $p_{+}(x)$ попеременно положительны (начиная с любой из них);

б) на $\Delta$ имеется ровно $n$ точек, в которых обе функции, $p_{-}(x)$ и $p_{+}(x)$, положительны, в остальных жсе точках обе они равны нулю.

ДокАзАтЕЛьство. Прежде всего отметим один простой частный случай: сушествует такая точка $c \in[-\infty,+\infty]$, что хотя бы с одной стороны от $c$ в любой близости от нее найдутся точки $x^{-}$и $x^{+}$(отличные от $c$ ), для которых $p_{-}\left(x^{-}\right)>0$, $p_{+}\left(x^{+}\right)>0$. Очевидно, в этом случае выполняется условие $\left.2.5, \mathrm{a}\right)$. Поэтому до конца доказательства мы будем считать, что для каждой точки $c \in\langle a, b\rangle$ существует такая ее окрестность, что с каждой стороны от $c$ в этой окрестности по крайней мере одна из функций $p_{-}(x), p_{+}(x)$ тождественно равна нулю (при этом окрестностью точки $-\infty$ называется любой промежуток $[-\infty, t)$ при $-\infty<t \leqslant+\infty$, а окрестностью точки $+\infty-$ любой промежуток $(t,+\infty]$ при $-\infty \leqslant t<+\infty)$.

Построим на $(-\infty,+\infty]$ некоторую специальную конечную последовательность $x_{1}<x_{2}<\cdots$ На множестве $[-\infty,+\infty] \backslash\langle a, b\rangle$, если оно не пусто, доопределим вес $p$ равенством $p_{-}(x)=p_{+}(x)=0$.

*1. Положим

$$
\begin{aligned}
& x_{1}^{-}=\sup \left\{x: p_{-}(t)=0 \quad \forall t \in[-\infty, x)\right\}, \\
& x_{1}^{+}=\sup \left\{x: p_{+}(t)=0 \quad \forall t \in[-\infty, x)\right\}, \\
& x_{0}=\min \left\{x_{1}^{-}, x_{1}^{+}\right\}, \quad x_{1}=\max \left\{x_{1}^{-}, x_{1}^{+}\right\} .
\end{aligned}
$$

Так как отмеченный выше простой случай заведомо имеет место, если промежуток $\langle a, b\rangle$ открыт слева, а $x_{1}=a$, то при открытом слева промежутке $\langle a, b\rangle$ будем считать, что $a<x_{1}$. При $x_{1}=+\infty$ построение последовательности $\left\{x_{k}\right\}$ заканчивается.

*2. Пусть $x_{1}<+\infty$. Тогда $x_{1} \leqslant b$, и если $x_{1}=b$, то $b \in\langle a, b\rangle$. Строим точку $x_{2}$. Возможны следующие два случая:

*2a. $x_{1}^{-} \neq x_{1}^{+}$

*2б. $x_{1}^{-}=x_{1}^{+}$.

В случае * $2 \mathrm{a}$, как мы увидим, построение последовательности $\left\{x_{k}\right\}$ приводит к утверждению 2.5, а), в случае же *2б оно приводит либо к утверждению 2.5, а), либо к 2.5, б).

Рассмотрим сначала случай $* 2 \mathrm{a}$, для определенности считая, что $x_{0}=$ $x_{1}^{+}<x_{1}^{-}=x_{1}$. Итак,

*2a. $x_{1}^{-} \neq x_{1}^{+}\left(x_{1}^{+}<x_{1}^{-}\right)$. Строим точку $x_{2}$. По сказанному вьше о простом частном случае теоремы при некотором $t>x_{1}$ хотя бы одна из функций $p_{-}$и $p_{+}$ тождественно равна нулю на интервале $\left(x_{1}, t\right)$. Разобьем случай $* 2$ а на подслучаи:

$* 2 \mathrm{a}_{+}$. если $p_{+}(x)=0$ на $\left(x_{1}, t\right)=\left(x_{1}^{-}, t\right)$, то положим

$$
x_{2}=\sup \left\{t: p_{+}(x)=0 \quad \forall x \in\left(x_{1}, t\right)\right\} ;
$$

* $2 \mathrm{a}_{-}$. если же при условии $* 2 \mathrm{a}$ случай $* 2 \mathrm{a}_{+}$не имеет места, то $p_{-}(x)=0$ на некотором интервале $\left(x_{1}, t\right)$ и $p_{-}\left(x_{1}\right)>0, p_{-}(x)=0$ при $x<x_{1}$. В этом случае положим

$$
x_{2}=\sup \left\{t: p_{-}(x)=0 \quad \forall x \in\left(x_{1}, t\right)\right\} .
$$


Итак, точка $x_{2}$ в случае ${ }^{*} 2$ а определена. Если $x_{2}=+\infty$, то построение точек $\left\{x_{k}\right\}$ заканчивается.

*3a. Пусть $x_{2}<+\infty$. Строим $x_{3}$. При некотором $t>x_{2}$ хотя бы одна из функций $p_{-}(x), p_{+}(x)$ тождественна нулю в интервале $\left(x_{2}, t\right)$.

* $3 \mathrm{a}_{+}$. Пусть имеет место случай $* 2 \mathrm{a}_{+}$, так что точка $x_{2}$ определена равенством $(2.5 ; 1)$, и пусть выполнено условие

${ }^{*} 3 \mathrm{a}_{+}^{-} \cdot p_{-}(x)=0$ на $\left(x_{2}, t\right)$ для некоторого $t>x_{2}$. Тогда положим

$$
x_{3}=\sup \left\{t: p_{-}(x)=0 \quad \forall x \in\left(x_{2}, t\right)\right\} .
$$

$* 3 \mathrm{a}_{+}^{+}$. Пусть имеет место случай $* 2 \mathrm{a}_{+}$, так что $x_{2}$ определена равенством $(2.5 ; 1)$, и условие $* 3 \mathrm{a}_{+}^{-}$не выполнено. Тогда при любом $t>x_{2}$ на интервале $\left(x_{2}, t\right)$ имеем $p_{-}(x) \not \equiv 0$, a $p_{+}(x) \equiv 0$ на некотором интервале $\left(x_{2}, t\right)$ и $p_{+}\left(x_{2}\right)>0$. В этом случае положим

$$
x_{3}=\sup \left\{t: p_{+}(x)=0 \quad \forall x \in\left(x_{2}, t\right)\right\} .
$$

${ }^{*} 3 \mathrm{a}_{-}$. Пусть случай ${ }^{*} 2 \mathrm{a}_{+}$не имеет места. Тогда при определении точки $x_{3}$ в приведенных в п. ${ }^{*} 3 \mathrm{a}_{+}$рассуждениях надо поменять ролями функции $p_{-}$и $p_{+}$. Если $x_{3}=+\infty$, процесс построения точек $\left\{x_{k}\right\}$ заканчивается, при $x_{3}<+\infty$ он продолжается.

Пусть уже определена точка $x_{k}$ при некотором $k \geqslant 2$. Определим точку $x_{k+1}$.

$*(k+1)$ a. Как всегда, при некотором $t>x_{k}$ хотя бы одна из функций $p_{-}, p_{+}$тождественно равна нулю на $\left(x_{k}, t\right)$. Сначала пусть интервал $\left(x_{k-1}, x_{k}\right)$ был определен как максимально широкий вправо от $x_{k-1}$ интервал, на котором тождественно равна нулю функция $p_{-}(x)$; такой интервал мы будем называть $(-)$-интервалом. Если при этом имеется такое $t_{+}>x_{k}$, что $p_{+}(x)=0$ на $\left(x_{k}, t_{+}\right)$, то интервал $\left(x_{k}, x_{k+1}\right)$ определим как самый широкий вправо от $x_{k}$ интервал, на котором $p_{+}(x) \equiv 0$. Такой интервал будем называть $(+)$-интервалом. Если же такого интервала $\left(x_{k}, t_{+}\right)$нет, то $\left(x_{k}, x_{k+1}\right)$ определим как $(-)$-интервал, т.е. как самый широкий вправо от $x_{k}$ интервал, на котором $p_{-}(x)=0$ (в этом случае, очевидно, $\left.p_{-}\left(x_{k}\right)>0\right)$. Если в этих выкладках всюду поменять местами индексы $(-)$ и $(+)$, то получим определение точки $x_{k+1}$ в случае, когда $\left(x_{k-1}, x_{k}\right)$ был определен как (+)-интервал.

Если $x_{k+1}=+\infty$, то процесс построения точек $x_{1}, x_{2}, x_{3}, \ldots$ заканчиваем, в противном случае продолжаем его. Заметим, что в случае $x_{k}=b \in\langle a, b\rangle$ и $x_{k+1}=+\infty$ интервалы $\left(x_{k-1}, x_{k}\right)$ и $\left(x_{k}, x_{k+1}\right)$ формально строятся как интервалы разных знаков. В связи с этим вспомним, что в случае, если концевая точка промежутка $\langle a, b\rangle$ является нулем некоторого элемента $l \in L$, то этому нулю элемента $l$ приписывается четность 1.

Пусть $x_{1}, x_{2}, x_{3}, \ldots$ - построенная таким образом последовательность точек. По сделанному в начале доказательства предположению можно считать эту последовательность конечной:

$$
x_{1}<x_{2}<x_{3}<\cdots<x_{j}<x_{j+1}=+\infty .
$$

Легко видеть, что $X=\left\{x_{i}\right\}_{1}^{j} \subset\langle a, b\rangle$ (об $x_{1}$ говорилось в начале доказательства; если бы точка $x_{j}$ не принадлежала промежутку $\langle a, b\rangle$, то было бы $x_{j}=+\infty$, что не так). 
Покажем, что в рассматриваемом случае выполнено утверждение 2.5 , а). Строим точки $\left\{a_{i}\right\}$.

Припишем точке $x_{i}, i=1, \ldots, j$, четность $n\left(x_{i}\right)=2$, если интервалы $\left(x_{i-1}, x_{i}\right)$ и $\left(x_{i}, x_{i+1}\right)$ либо оба являются $(-)$-интервалами, либо оба являются $(+)$-интервалами. В противном случае точке $x_{i}$ припишем четность $n\left(x_{i}\right)=1$. В частности, $n\left(x_{1}\right)=1$ в случае ${ }^{*} 2 \mathrm{a}_{+}$и $n\left(x_{1}\right)=2$ в случае ${ }^{*} 2 \mathrm{a}_{-}, n\left(x_{j}\right)=1$ при $x_{j}=b \in\langle a, b\rangle$.

Покажем, что

$$
m:=n\left(x_{1}\right)+n\left(x_{2}\right)+\cdots+n\left(x_{j}\right) \geqslant n .
$$

Действительно, если бы было $m<n$, то по лемме 2.4 существовал бы полином $l \in L$, имеющий нули в точках $x_{i}$ с четностями $n\left(x_{i}\right)$ соответственно $(i=1, \ldots, j)$, причем $l(x)$ мог бы иметь еще разве лишь один (нечетный) нуль на множестве $\langle a, b\rangle \backslash(a, b) \backslash X$. При этом один из полиномов $l(x),-l(x)$ обладает следуюшим свойством: на всех $(-)$-интервалах $\left(x_{i-1}, x_{i}\right)$ он отрицателен, на всех $(+)$-интервалах положителен, равен нулю во всех точках $x_{i}$. Поэтому при соответствующем выборе знака числа $y$ имеем

$$
|y l|_{p,\langle a, b\rangle}=0, \quad\|y l\|_{F} \geqslant|y| \cdot\left|l\left(x^{*}\right)\right|,
$$

где $x^{*}$ - любая точка из $F$, в которой $l\left(x^{*}\right)$ отличен от нуля. Такая точка существует. Действительно, при $m=n-1$ это любая точка множества $F \backslash X$ (в этом случае разность $n-m$ нечетна и $l(x) \neq 0$ на множестве $F \backslash X$, содержащем не менее $n-j \geqslant n-m=1$ точек; см. лемму 2.4$)$. При $m \leqslant n-2$ это либо любая точка множества $F \backslash X$ (содержащего в этом случае не менее $n-j \geqslant n-m \geqslant n-(n-2)=2$ точек), если $\langle a, b\rangle$ не содержит своих конщевых точек, либо любая точка этого множества, кроме разве лишь одной конщевой точки промежутка $\langle a, b\rangle$ (см 2.4). Устремляя $|y| \mathrm{K}+\infty$ при сохранении знака $y$, из $(2.5 ; 6)$ получаем $W(p, L, F)=\infty$. Противоречие с условием доказываемой теоремы. Этим неравенство $m \geqslant n$ доказано.

Так как $x_{1}^{+}$отлично от $x_{1}^{-}$, именно $x_{1}^{+}<x_{1}^{-}=x_{1}$, то на $\langle a, b\rangle$ найдется такая точка $a_{1}<x_{1}$, что $p_{+}\left(a_{1}\right)>0$. Если $n\left(x_{1}\right)=1$, то найдется такая точка $a_{2} \in\left[x_{1}, x_{2}\right)$, что $p_{-}\left(a_{2}\right)>0$. Если же $n\left(x_{1}\right)=2$, то полагаем $a_{2}=x_{1}$. Тогда $p_{-}\left(a_{2}\right)=p_{-}\left(x_{1}\right)>0$, и найдется такая точка $a_{3} \in\left(x_{1}, x_{2}\right)$, что $p_{+}\left(a_{3}\right)>0$, и т.д. При этом каждая точка $x_{i}$ с $n\left(x_{i}\right)=1$ дает ровно одну точку $a_{k}$, а точка $x_{i}$ с $n\left(x_{i}\right)=2$ дает две различные точки $a_{k}$, одна из которых совпадает с этой точкой $x_{i}$. Всего мы получим $m+1 \geqslant n+1$ точек $a_{k}$, в которых $p_{+}(x)$ и $p_{-}(x)$ попеременно положительны. Этим доказано, что в случае $* 2$ а справедливо утверждение $2.5, \mathrm{a})$.

*2аб. Пусть теперь $x_{1}^{-}=x_{1}^{+}=x_{1}$. Положим

$$
\begin{aligned}
& x_{2}^{-}=\sup \left\{t: t>x_{1}, p_{-}(x)=0 \quad \forall x \in\left(x_{1}, t\right)\right\}, \\
& x_{2}^{+}=\sup \left\{t: t>x_{1}, p_{+}(x)=0 \quad \forall x \in\left(x_{1}, t\right)\right\}, \quad x_{2}=\max \left\{x_{2}^{-}, x_{2}^{+}\right\}
\end{aligned}
$$

(в частности, если, например, $p_{-}(x)$ не тождественна нулю на любом интервале $\left(x_{1}, t\right)$, то считаем $\left.x_{2}^{-}=x_{1}\right)$.

Если $x_{2}=+\infty$, то процесс построения точек $x_{k}$ заканчивается. Если $x_{2}<+\infty$ и $x_{2}^{+}$отлична от $x_{2}^{-}$(для определенности пусть $x_{2}^{+}<x_{2}^{-}=x_{2}$ ), то продолжим 
построение точек $x_{k}$ так, как это указано в п. ${ }^{*} 2 \mathrm{a}$ (с той лишш разницей, что роль точки $x_{1}$ теперь будет играть точка $x_{2}$, а роль $x_{2}$ - точка $\left.x_{3}\right)$. Как и вьше, устанавливается, что число $m$ точек $x_{1}, x_{2}, \ldots, x_{j}$ с учетом их четности не меньше $n, \mathrm{a}$ число точек $a_{k}$, в которых функции $p_{-}(x)$ и $p_{+}(x)$ попеременно положительны, не менее $n+1$ ( $a_{1}=x_{1}, p_{-}\left(a_{1}\right)>0, a_{2} \in\left(x_{1}, x_{2}\right), p_{+}\left(a_{2}\right)>0$ и т.д.).

Если в процессе построения точек $x_{1}, x_{2}, \ldots, x_{j}$ при некотором $i$ окажется, что $x_{i}^{-}$отлична от $x_{i}^{+}$, то получим случай 2.5, а) теоремы. Если же при всех $i=$ $1,2, \ldots, j$ имеем $x_{i}^{-}=x_{i}^{+}$, то $j \geqslant n$. Действительно, если бы было $j<n$, то по лемме 2.4 нашелся бы полином $l \in L$ с нечетными нулями в точках $x_{1}, x_{2}, \ldots, x_{j}$, для которого, как и в случае $2 \mathrm{a}$, получили бы для чисел $y$ определенного знака равенство $|y l|_{p,\langle a, b\rangle}=0$, в то время как $\|y l\| \rightarrow \infty$ при $|y| \rightarrow \infty$. Отсюда $W(p, L, F)=\infty$. Итак, $j \geqslant n$. Очевидно, при $j=n$ справедливо утверждение 2.5, б), в случае же $j \geqslant n+1-$ утверждение 2.5, a). Теорема доказана.

Приступая к доказательству достаточности условий 2.5, a) и 2.5, б) для конечности $W(p, L, F)$, установим еще одно свойство полиномов по чебышевской системe.

2.6. ЛЕммА. Пусть $L-n$-мерное чебышевское подпространство на промежутке $\langle a, b\rangle, \quad a_{1}<a_{2}<\cdots<a_{n+1}-$ точки из $\langle a, b\rangle ;$ при каждом $q=1, \ldots, n+1$ nусть $_{q}(x) \in L$, причем $l_{q}\left(a_{i}\right)=(-1)^{i-1}$ при всех $i=1, \ldots, n+1$, $i \neq q$. Тогда если $l \in L u$

$$
l\left(a_{1}\right) \leqslant 1, \quad l\left(a_{2}\right) \geqslant-1, \quad l\left(a_{3}\right) \leqslant 1, \quad l\left(a_{4}\right) \geqslant-1, \quad \ldots,
$$

то при каждом $x \in\langle a, b\rangle$ имеем неравенство

$$
\min \left\{l_{q}(x): 1 \leqslant q \leqslant n+1\right\} \leqslant l(x) \leqslant \max \left\{l_{q}(x): 1 \leqslant q \leqslant n+1\right\} .
$$

ДокАЗАТЕльство. Не ограничивая обшности, будем считать все неравенства $(2.6 ; 1)$ строгими (так как вместо $l(x)$ можно рассмотреть $t l(x)$ при $0<t=$ const $<1$, а затем устремить $t$ к 1 ). Нетрудно видеть, что при нечетном $n$ выполняются условия:

1) $l(x) \leqslant l_{n+1}(x)$ при $x \in\left\langle a, a_{1}\right]$;

2) $l(x) \leqslant l_{2 j}(x)$ при $x \in\left[a_{2 j-1}, a_{2 j+1}\right], j=1, \ldots,[n / 2]$;

3) $l(x) \leqslant l_{n+1}(x)$ при $x \in\left[a_{n}, b\right\rangle$.

Если бы было, например, $l\left(x_{0}\right)>l_{2 j}\left(x_{0}\right)$ при некотором $x_{0}$ из $\left[a_{2 j-1}, a_{2 j+1}\right]$, то $x_{0} \in\left(a_{2 j-1}, a_{2 j+1}\right)$ и графики функций $l(x)$ и $l_{2 j}(x)$ пересеклись бы по крайней мере дважды над $\left(a_{2 j-1}, a_{2 j+1}\right)$ и по меньшей мере еще по одному разу над каждым из $n-2$ интервалов $\left(a_{i}, a_{i+1}\right)$ с $i=1, \ldots, n, i \neq 2 j-1,2 j$. Отсюда следует, что элемент $l(x)-l_{2 j}(x)$ имеет не менее $n$ нулей на $\langle a, b\rangle$, т.е. $l(x) \equiv l_{2 j}(x)$, что невозможно.

Аналогично проверяется, что в случае четного $n$ выполняются неравенства 1 ) и 2), а вместо 3 ) - неравенство

$\left.3^{\prime}\right) l(x) \leqslant l_{1}(x)$ при $x \in\left[a_{n+1}, b\right\rangle$.

Так же доказывается и другая серия неравенств:

4) $l(x) \geqslant l_{1}(x)$ при $x \in\left\langle a, a_{2}\right]$;

5) $l(x) \geqslant l_{2 j+1}(x)$ при $x \in\left[a_{2 j}, a_{2 j+2}\right], j=1, \ldots,[(n-1) / 2]$; 
6) $l(x) \geqslant l_{n+1}(x)$ при четном $n$ и $x \in\left[a_{n}, b\right\rangle$;

$\left.6^{\prime}\right) l(x) \geqslant l_{1}(x)$ при нечетном $n$ и $x \in\left[a_{n+1}, b\right\rangle$.

Доказательство закончено.

Следуюшая теорема является центральной в этом параграфе.

2.7. ТеОрема. Пусть $L-n$-мерное чебышевское подпространство на промежутке $\Delta=\langle a, b\rangle,-\infty \leqslant a<b \leqslant+\infty$, порожденное некоторой чебышевской системой $\left\{\varphi_{k}\right\}_{k=1}^{n}, \quad F \subset \Delta$, и выполнено хотя бы одно из условий:

1) все функиии системы $\left\{\varphi_{k}\right\}$ ограничены на $\Delta$;

2) $F$ - компактная часть промежутка $\Delta$, состоящая не менее чем из $n$ точек.

Тогда, каков бы ни был знакочувствительный вес р (не обязательно конечньй ) на промежутке $\Delta$, выполнение одного и только одного из условий 2.5, a) или 2.5, б) необходимо и достаточно для конечности свободы $W(p, L, F)$.

ДокАзАтельСтво. Необходимость уже установлена теоремой 2.5. Достаточность будем доказывать лишь для случая конечного веса $p$, так как с уменьшением веса свобода лишш увеличивается (см. свойство 6) в п. 2.0).

Пусть сначала вьполняется условие 2.5, a).

Для определенности пусть $p_{+}\left(a_{1}\right)>0, p_{-}\left(a_{2}\right)>0, p_{+}\left(a_{3}\right)>0, \ldots$ Тогда для любого $v \in L \quad\left(v(x)=\sum c_{k} \varphi_{k}(x)\right)$, для которого $|v|_{p, \Delta} \leqslant 1$, будет

$$
v\left(a_{1}\right) \cdot p_{+}\left(a_{1}\right) \leqslant 1, \quad v\left(a_{2}\right) \cdot p_{-}\left(a_{2}\right) \geqslant-1, \quad v\left(a_{3}\right) \cdot p_{+}\left(a_{3}\right) \leqslant 1
$$

и т.д. Поэтому при $r=\min \left\{p_{+}\left(a_{1}\right), p_{-}\left(a_{2}\right), p_{+}\left(a_{3}\right), \ldots\right\}, r>0$, функция $l(x)=$ $r v(x)$ удовлетворяет неравенствам $(2.6 ; 1)$. По лемме 2.6

$$
\begin{aligned}
m(x) & :=\frac{1}{r} \min \left\{l_{q}(x): 1 \leqslant q \leqslant n+1\right\} \leqslant v(x) \leqslant M(x) \\
& :=\frac{1}{r} \max \left\{l_{q}(x): 1 \leqslant q \leqslant n+1\right\}
\end{aligned}
$$

при всех $x \in \Delta$, где функции $m(x)$ и $M(x)$ не зависят от $v$. Эти функции, как и все $\varphi_{k}$, ограничены на $F$ (см. условия 1 ) и 2) доказываемой теоремы), так что $\|v\|_{F} \leqslant M_{1}=$ const $<\infty$ независимо от функции $v \in L$, лишь бы было $|v|_{p, \Delta} \leqslant 1$.

Рассмотрим теперь случай выполнения условия 2.5, б). Положим

$$
R:=\min \left\{p_{-}\left(a_{1}\right), p_{+}\left(a_{1}\right), p_{-}\left(a_{2}\right), p_{+}\left(a_{2}\right), \ldots\right\}, \quad 0<R<\infty .
$$

Если $l \in L$ и $|l|_{p, \Delta}=\infty$, то $\|l\|_{F} /|l|_{p, \Delta}=0$. Если $|l|_{p, \Delta}<\infty$, имеем очевидное неравенство

$$
\left|l\left(a_{j}\right)\right| \leqslant R^{-1} \cdot|l|_{p, \Delta} \quad \forall l \in L .
$$

При каждом $j=1, \ldots, n$ обозначим через $l_{j}(x)$ интерполяционньй полином по системе $\left\{\varphi_{k}\right\}$, равный единице в точке $a_{j}$ и равный нулю во всех остальных точках $a_{i}$ (см. $\left[14\right.$, с. 21]). Обозначим через $S$ сумму по $j=1, \ldots, n$ норм $\left\|l_{j}\right\|_{F}(S<\infty)$. Если $l \in L$, то

$$
l(x)=l\left(a_{1}\right) l_{1}(x)+l\left(a_{2}\right) l_{2}(x)+\cdots+l\left(a_{n}\right) l_{n}(x),
$$


так что (см. $(2.7 ; 1))$

$$
\|l\|_{F} \leqslant R^{-1} \cdot|l|_{p, \Delta} \cdot S, \quad \frac{\|l\|_{F}}{|l|_{p, \Delta}} \leqslant \frac{S}{R} \quad \forall l \in L, \quad W(p, L) \leqslant \frac{S}{R} .
$$

Доказательство закончено.

Сделаем одно простое замечание, которое нам понадобится в дальнейшем.

2.7а; 0. Напомним, что (замкнутыцм) носителем $\operatorname{supp}(f)$ функции $f$ называется множество всех точек $t \in[-\infty,+\infty]$, у которых в любой окрестности имеются точки $x$, в которых $f(x) \neq 0$. Разумеется, если $t \in \operatorname{supp}(f)$ - предельная точка для $\operatorname{supp}(f)$, то может быть $f(t)=0$. Носитель $\operatorname{supp}(p)$ и ядро П $(p)$ веса $p=\left(p_{-}, p_{+}\right)$ определим так:

$$
\operatorname{supp}(p):=\operatorname{supp}\left(p_{-}\right) \cup \operatorname{supp}\left(p_{+}\right), \quad \Pi(p):=\operatorname{supp}\left(p_{-}\right) \cap \operatorname{supp}\left(p_{+}\right) .
$$

2.7a. СлЕДСТВИЕ. Если вес $p=\left(p_{-}, p_{+}\right)$(не обязательно конечный) на промежутке $\Delta$ таков, что $\Pi(p)$ содержит не менее $n \geqslant 1$ точек, то имеет место один из случаев 2.5, а) или 2.5, б), так что если при этом $\Delta$ замкнутый отрезок, $L-n$-мерное чебышевское подпространство на $\Delta$, то $W(p, L)<\infty$ (последнее - по теореме 2.7$)$.

\section{§3. Случай произвольного семейства ограниченных функций}

Если $L$ - произвольное конечномерное линейное пространство функций, определенных на $E \cup F$ и ограниченных на $F$, то достаточные условия конечности свободы $W(p, L, F)$ (положительности жесткости $S(p, L, F)$ ) уже не могут быть сформулированы лишь в терминах свойств веса $p$ и линейной размерности пространства $L$. Для простоты считая $F=E$, укажем условие конечности $W(p, L, F)$ для произвольного семейства ограниченных функций (теорема 2.12). Этот результат содержится в более общем утверждении 2.11 .

2.8. Введем необходимые обозначения и определения. Пусть $L-$ некоторое семейство ограниченных функций на $E \subset \mathbb{R}$, не сводящееся к единственной функции $l(x) \equiv 0$, функция $f$ ограничена на $E$.

1) $\omega(f, t)=\omega(f, E, t):=\sup \left\{\left|f\left(x^{\prime}\right)-f\left(x^{\prime \prime}\right)\right|: x^{\prime}, x^{\prime \prime} \in E,\left|x^{\prime}-x^{\prime \prime}\right| \leqslant t\right\}-$ модуль непрерывности функции $f$ на множестве $E(t \geqslant 0)$. Если вес $p=\left(p_{-}, p_{+}\right)$ определен на $E$, то величину

1a) $\omega(p, t):=\max \left\{\omega\left(p_{-}, t\right), \omega\left(p_{+}, t\right)\right\}$ назовем его модулем непрерывности на множестве $E$. Если справа в 1$)$ вместо $f(x)$ взять $f^{-}(x)=\max \{-f(x), 0\}$ или $f^{+}(x)=\max \{f(x), 0\}$, то получим определения величин $\omega_{-}(f, t)$ и $\omega_{+}(f, t)$ соответственно. Очевидно,

$$
\max \left\{\omega_{-}(f, t), \omega_{+}(f, t)\right\} \leqslant \omega(f, t) \leqslant \omega_{-}(f, t)+\omega_{+}(f, t) \leqslant 2 \omega(f, t) .
$$

2) $\omega(L, t)=\omega(L, E, t):=\sup \{\omega(l /\|l\|, E, t): l \in L, l(x) \not \equiv 0\}, t \geqslant 0,-$ модуль непрерывности семейства $L$. Замена в этом определении $\omega$ на $\omega_{-}$или $\omega_{+}$дает определение величин $\omega_{-}(L, t)=\omega_{-}(L, E, t)$ и $\omega_{+}(L, t)=\omega_{+}(L, E, t)$ соответственно.

При $t \geqslant 0$ назовем величину 
3) $\omega_{s g}(L, t)=\omega_{s g}(L, E, t)=\max \left\{\omega_{-}(L, E, t), \omega_{+}(L, E, t)\right\}$ знакочувствительным.м модулем непрерывности семейства L. Очевидно

$$
\frac{\omega(L, t)}{2} \leqslant \omega_{s g}(L, t) \leqslant \omega(L, t) .
$$

Если $p, q$ - знакочувствительные веса на $E, L \subset M(E)$, положим

4) $\underline{\sigma}(p, q, L ; t):=\min \{\|p\|,\|q\|\} \cdot \omega_{s g}(L, t+0)+t, t \geqslant 0$ (как обычно, $g\left(t_{0}+0\right)$ обозначает предел функции $g(t)$ при стремлении $t$ к $t_{0}$ справа).

Если $\mathscr{L}$ - линейное пространство, $f \in \mathscr{L}$ и $L \subset \mathscr{L}$, положим

5) $L(f):=\{l-f: l \in L\}$,

6) dif $L:=\{g-h: g, h \in L\}$.

Очевидно, в случае линейного пространства $L$ имеем $\operatorname{dif} L=L$.

Следующие две леммы касаются характера изменения $\omega(L, t)$ после сужения области определения семейства $L$ и после параллельного сдвига этого семейства.

2.9. Лемма. Пусть $\Delta=\langle a, b\rangle \subset \mathbb{R}, L \subset C(\Delta)$, промежуток $J$ лежит на $\Delta, \quad h:=h(J, \Delta)$ - хаусдорфово расстояние между $J u \Delta, \omega(L, h)<1$, $L[J]$ - сужение семейства $L$ на промежуток $J$. Тогда

$$
\omega(L[J], t) \leqslant \frac{\omega(L, t)}{1-\omega(L, h)}, \quad t \geqslant 0
$$

ДокАЗАТЕЛЬСТво. Так как $\|l\|_{J}+\omega(l, h) \geqslant\|l\|_{\Delta}$ для любого $l \in L \quad$ и $\omega(L, h)<1$, то $\|l\|_{\Delta} /\|l\|_{J} \leqslant 1 /(1-\omega(L, h))$. Поэтому

$$
\omega\left(\frac{l}{\|l\|_{J}}, J, t\right) \leqslant \omega\left(\frac{l}{\|l\|_{\Delta}}, \Delta, t\right) \cdot\left(\frac{\|l\|_{\Delta}}{\|l\|_{J}}\right) \leqslant \frac{\omega(L, t)}{1-\omega(L, h)}
$$

А это эквивалентно неравенству $(2.9 ; 1)$.

Напомним, что $E(L, f)$ обозначает наименьшее уклонение множества $L$ из нормированного пространства $\mathscr{L}$ от $f \in \mathscr{L}$ по норме $\mathscr{L}$.

2.10. Лемма. Пусть $E \subset \mathbb{R}, L \subset M(E), f \in M(E), E(L, f)>0, \varepsilon>0$, $l$ - любая функция из $L$, для которой $\|l-f\|_{E} \leqslant E(L, f)+\varepsilon$. Тогда

$$
\frac{\omega(l-f, t)}{E(L, f)+\varepsilon} \leqslant \omega(L(f), t) \leqslant \frac{\omega(l-f, t)}{E(L, f)}+\left(2+\frac{\varepsilon}{E(L, f)}\right) \omega(L, t) \quad \forall t>0 .
$$

2.10a. ЗАмЕЧАнИЕ. Появление $\varepsilon>0$ в этой формулировке связано с тем, что $f$ может не иметь элемента наилучшего приближения. Если же $l=l(L, f)$, то в $(2.10 ; 1)$ можно положить $\varepsilon=0$. 
ДоКАЗАТЕЛЬСТВо. Левое из неравенств $(2.10 ; 1)$ очевидно. С другой стороны, если $\eta>0$ и функция $m \in L$ такова, что

$$
\omega\left(\frac{m-f}{\|m-f\|}, t\right)>\omega(L(f), t)-\eta,
$$

Tо

$$
\begin{gathered}
\|m-f\| \geqslant E(L, f) \geqslant\|l-f\|-\varepsilon, \\
\omega(L(f), t)-\eta<\omega\left(\frac{m-f}{\|m-f\|}, t\right) \leqslant \frac{\omega(l-f, t)}{\|m-f\|}+\frac{\|m-l\|}{\|m-f\|} \omega\left(\frac{m-l}{\|m-l\|}, t\right) \\
\leqslant \frac{\omega(l-f, t)}{\|m-f\|}+\frac{\|m-f\|+\|f-l\|}{\|m-f\|} \omega(L, t) \\
\leqslant \frac{\omega(l-f, t)}{E(L, f)}+\left(2+\frac{\varepsilon}{E(L, f)}\right) \omega(L, t),
\end{gathered}
$$

что и требовалось доказать.

Приводимые ниже теоремы 2.11 и 2.12 касаются условий конечности свободы $W(p, L)$.

2.11. ТеОрема. Пусть $L \subset M(E), E \subset \mathbb{R}, p$ и $q$-ограниченные знакочувствительные веса на $E, d=d(p, q)$. Тогда если $W(q, L)<\infty u$

$$
\max \left\{\|q\| \omega_{s g}(L, d+0), d\right\}<S(q, L),
$$

mo $u W(p, L)<\infty$.

2.11a. ЗАмечАнИЕ. Для конечности $W(p, L)$ достаточно, чтобы неравенство $(2.11 ; 1)$ выполнялось при замене $p$ на $C_{1} p$ и $q$ на $C_{2} q$ (соответственно $d$ - на $\left.d\left(C_{1} p, C_{2} q\right)\right)$, где $C_{1}$ и $C_{2}$ - какие-либо положительные постоянные. Отметим также, что условие $(2.11 ; 1)$ в определенном смысле неулучшаемо; см. ниже замечание 2.12a.

ДокАЗАТЕЛЬСТво. Предположим, что

$$
S(p, L)=0 \quad \text { при } \quad\|q\| \omega_{s g}(L, d+0)<S(q, L) \quad \text { и } \quad d<s(q, L) .
$$

Тогда, каким бы ни было $s>0$, существует нетождественная нулю функция $v \in L$, для которой $|v|_{p, E} /\|v\|_{E}<s^{2}\|q\| / 3$. Для $l=v /\|v\|_{E}$ имеем

$$
|l|_{p, E}<\frac{s^{2}\|q\|}{3}
$$

Положим также

$$
w=W(q, L), \quad t=\min \left\{1-w d, 1-w \cdot\|q\| \omega_{s g}(L, d+0)\right\} .
$$

По условию теоремы $t>0$. Пусть теперь $s>0$ таково, что

$$
s \cdot\|q\| \cdot w<t .
$$


Обозначим через $K$ множество всех тех $x \in E$, при которых

$$
\frac{|(l, q)(x)|}{|l|_{q, E}}>1-t+s \cdot\|q\| \cdot w .
$$

Тогда при $x \in K$

$$
|l(x)|>\frac{|l|_{q, E}}{\|q\|} \cdot(1-t+s \cdot\|q\| \cdot w), \quad x \in K .
$$

При $x \in K$ также будет одно из двух: либо

$$
l(x)<0, \quad q_{-}(x)>d+s\|q\|,
$$

либо

$$
l(x)>0, \quad q_{+}(x)>d+s\|q\|
$$

(действительно, в противном случае по определению $w$ из неравенств $w d \leqslant 1-t$ (см. $(2.11 ; 4))$ и $(2.11 ; 6)$ имеем для некоторого $x \in K$ неравенство

$$
\frac{|(l, q)(x)|}{|l|_{q, E}} \leqslant \frac{\|l\|(d+s\|q\|)}{|l|_{q, E}} \leqslant w(d+s\|q\|) \leqslant 1-t+s w\|q\|<\frac{|(l, q)(x)|}{|l|_{q, E}}
$$

явно противоречивое).

Пусть $x \in K$,

$$
d^{\prime} \in\left(d, d+\frac{s}{3}\|q\|\right)
$$

и пусть для определенности $l(x)>0$. По определению расстояния $d=d(p, q)$ найдется такая точка $v \in E$, что $|x-v| \leqslant d^{\prime}, p_{+}(v) \geqslant q_{+}(x)-d^{\prime}$, откуда (см. $(2.11 ; 9),(2.11 ; 10))$

$$
p_{+}(v) \geqslant\left(q_{+}(x)-d\right)-\left(d^{\prime}-d\right)>s\|q\|-\frac{s}{3}\|q\|=\frac{2 s}{3}\|q\| .
$$

Отсюда и из $(2.11 ; 3)$ имеем

$$
l^{+}(v) \leqslant \frac{|l|_{p, E}}{p_{+}(v)} \leqslant \frac{s^{2}\|q\|}{3 p_{+}(v)} \leqslant \frac{s}{2} .
$$

Очевидно, $\omega_{s g}\left(L, d^{\prime}\right) \geqslant l^{+}(x)-l^{+}(v)$. Из этого неравенства, из неравенств $(2.11 ; 7)$ и $(2.11 ; 11)$, равенства $\|l\|=1$, определения жесткости $S(q, L)=1 / w$ и соотношения $(2.11 ; 4)$ получаем

$$
\begin{aligned}
\omega_{s g}\left(L, d^{\prime}\right) & >\frac{|l|_{q, E}}{\|q\|}(1-t+s \cdot\|q\| \cdot w)-\frac{s}{2} \geqslant S(q, L) \frac{1-t}{\|q\|}+s S(q, L) w-\frac{s}{2} \\
& =\frac{1-t}{w\|q\|}+\frac{s}{2} \geqslant \omega_{s g}(L, d+0)+\frac{s}{2} .
\end{aligned}
$$

Переходя здесь к пределу при $d^{\prime} \rightarrow d$ (при фиксированном $s$ ), получим противоречивое неравенство $\omega_{s g}(L, d+0)>\omega_{s g}(L, d+0)$. Итак, предположение о том, что $S(p, L)=0$, неверно, и теорема доказана. 
2.12. Теорема. Пусть $L \subset M(E), \quad E \subset \mathbb{R}, \quad p$ - нетривиальный ограниченныи иес на $E, D=\max \left\{d_{-}, d_{+}\right\}$, где $d_{-}, d_{+}-$хаусдорфовы расстояния между $E$ и носителями $\operatorname{supp}\left(p_{-}\right), \operatorname{supp}\left(p_{+}\right)$функиий $p_{-}, p_{+}$соответственно. Тогда если

$$
\omega_{s g}(L, D+0)<1
$$

(в частности, если $\omega(L, D+0)<1)$, то $W(p, L)<\infty$ u "p-норма" $|\cdot|_{p, E}$ эквивалентна равномерной норме $\|\cdot\|_{E}$.

ДокАЗАТЕЛЬСтво. Не ограничивая общности, считаем, что $\|p\|=C$, где $C$ наперед заданная положительная постоянная. Определим знакочувствительньй вес $q$ равенством $q=(C, C)$. Тогда $S(q, L)=C$ и $d=d(p, q)<C$ при $C>D$. Последнее вытекает из следующих фактов.

$1^{\circ}$. Расстояние Минковского между точкой $(x, C)$ на графике функции $q_{-}$ (или $q_{+}$) и дополненным графиком функции $p_{-}\left(\right.$соответственно $\left.p_{+}\right)$является непрерывной функцией от $x$ на замыкании $\bar{E}$ множества $E$.

$2^{\circ}$. Если $x$ лежит на $\bar{E}$, то $C$-окрестность Минковского каждой точки $(x, C)$ содержит точки графика функции $p_{-}(x)$, так что расстояние Минковского между $(x, C)$ и дополненным графиком функции $p_{-}(x)$ строго меньше $C$.

По $1^{\circ} C^{\prime}<C$, где $C^{\prime}$ - максимум по $x$ этих расстояний на $\bar{E}$. Поэтому график функции $\max \left\{p_{-}(x), q_{-}(x)\right\} \equiv C$ находится в замкнутой $C^{\prime}$-окрестности Минковского дополненного графика функции $p_{-}(x)$. Аналогичное заключение с некоторой постоянной $C^{\prime \prime}<C$ получаем и для $p_{+}$.

Итак, $d(p, q)=\max \left\{C^{\prime}, C^{\prime \prime}\right\}<C$. Отсюда и из условия $(2.12 ; 1)$ следует, что при $C$, достаточно близком к $D$, выполняется условие $(2.11 ; 1)$ теоремы 2.11 , из которой и следует теорема 2.12 .

2.12a. ЗАмЕчАниЕ. Каким бы ни был нетривиальный ограниченный знакочувствительный вес $p(x), x \in E=\Delta=[a, b]$, условие $(2.12 ; 1)$ нельзя ослабить даже на классе семейств $L$, состояших из непрерывных ограниченных функций $(L \subset C(\Delta))$.

Действительно, пусть $p=\left(p_{-}, p_{+}\right)$- произвольный ограниченный вес на $\Delta, D$ величина, определенная в теореме $2.12, D>0$. Тогда на $\Delta$ имеется интервал $(r, s)$ длины $2 D$, дополнительный либо к $\operatorname{supp}\left(p_{-}\right)$, либо к $\operatorname{supp}\left(p_{+}\right)$. Для определенности пусть $(r, s)$ - дополнительный интервал к $\operatorname{supp}\left(p_{+}\right)$. Возьмем на $\Delta$ какую-либо непрерывную функцию $v(x)$, равную 0 вне $(r, s)$, положительную на $(r, s)$ и принимающую строгий абсолютный максимум в середине этого интервала. Тогда $|v|_{p, \Delta}=0$, так что для любого семейства $L$, содержашего $v$, имеем $S(p, L)=0$. При этом $\omega_{s g}(L, D) \geqslant \omega(v /\|v\|, D)=1$, и если, например, $L$ состоит из функций вида $C v(x), C=$ const $\in \mathbb{R}$, то $\omega_{s g}(L, t)<1$ при $t<D$.

2.12б. ЗАмЕчАнИЕ. Если $L$ - чебышевское подпространство линейной размерности $\geqslant 2$ на промежутке $E=\langle a, b\rangle$, то достаточное условие $(2.12 ; 1)$ конечности свободы $W(p, L)$ является существенно более узким, чем соответствующие достаточные условия 2.5, a) или 2.5, б) теоремы 2.7 . Однако и в этом случае условие $(2.12 ; 1)$ остается, вообше говоря, неулучшаемым.

Первая часть этого утверждения следует из необходимости условий 2.5, a) и 2.5, б) (теорема 2.7 ) и того, что для любого знакочувствительного веса $p$, 
удовлетворяющего одному из этих условий, а также условию $D \geqslant(b-a) / 2$ (определение $D$ см. в теореме 2.12), всегда имеем $\omega_{s g}(L, D) \geqslant 1$ (ведь $\omega_{s g}(l /\|l\|,(b-a) / 2)=1$ для любой функции $l \in L$, имеющей нуль в середине $\langle a, b\rangle, l \neq \equiv 0)$. Таким образом, в этом случае теорема 2.7 работает, а теорема 2.12 нет.

Для доказательства неулучшаемости условия $(2.12 ; 1)$ на классе чебышевских подпространств рассмотрим семейство $L$ тригонометрических полиномов порядка не выше $n \geqslant 1$ на множестве $E=[-\pi, \pi)$. Нетрудно видеть, что $\omega_{s g}(L, t)=\sin n t$ при $0 \leqslant t \leqslant \pi /(2 n)$ и что $\omega_{s g}(L, t)=1$ при $t \geqslant \pi /(2 n)$. Если $p(x)=(1,1)$ при $x=\pi(2 k+1) /(2 n)$, где $k=-n, \ldots,-1,0,1, \ldots, n-1$, и $p(x) \equiv(0,0)$ в остальных точках $E$, то $|\cos n x|_{p, E}=0, D=\pi /(2 n)$. Поэтому $S(p, L)=0, \omega_{s g}(L, t)<1$ при $t<D, \omega_{s g}(L, D)=1$.

Следующая теорема оценивает свободу системы $(P ; L(f))$, где $L(f)$ - параллельный перенос множества $L(f \in \mathscr{L})$; см. п. 2.8, 5).

2.13. ТЕОРЕМА. Пусть $P$ - ограниченный сублинейный функционал на линейном нормированном пространстве $\mathscr{L}, L$-множество из $\mathscr{L}, f \in \mathscr{L}$, $E(P ; L, f)>0$. Тогда

$$
W(P ; L(f)) \leqslant 3\|P\| \frac{E(L, f)}{E(P ; L, f)} \cdot W(P ; \operatorname{dif} L) .
$$

В частности, если $L$ - линейное подпространство из $\mathscr{L}$, то

$$
W(P ; L(f)) \leqslant 3\|P\| \frac{E(L, f)}{E(P ; L, f)} W(P ; L) .
$$

ДоказАтельство. Считаем $W(P ; \operatorname{dif} L)<\infty$, поскольку в противном случае неравенство $(2.13 ; 1)$ тривиально. Пусть $s>0$ и вектор $m \in L$ таков, что $\| f-$ $m \|<E(L, f)+s$. Тогда при $r:=E(P ; L, f)$ имеем для любого $l \in L$

$$
\begin{aligned}
\frac{\|l-f\|}{P(l-f)} & \leqslant \frac{\|l-m\|}{P(l-f)}+\frac{\|m-f\|}{P(l-f)} \leqslant \frac{W(P ; \operatorname{dif} L) P(l-m)}{P(l-f)}+\frac{\|m-f\|}{P(l-f)} \\
& \leqslant W(P ; \operatorname{dif} L)\left(1+\frac{P(f-m)}{P(l-f)}\right)+\frac{\|m-f\|}{P(l-f)} \\
& \leqslant W(P ; \operatorname{dif} L)\left(1+\frac{(E(L, f)+s)\|P\|}{r}\right)+\frac{E(L, f)+s}{r} .
\end{aligned}
$$

Отсюда, учитьвая произвольность $s>0$ и $l \in L$, а также неравенства $1 \leqslant$ $\|P\| E(L, f) / r$ и $1 \leqslant\|P\| W(P ; \operatorname{dif} L)($ см. $(2.0 ; 2))$, получаем

$$
\begin{aligned}
W(P ; L(f)) & \leqslant W(P ; \operatorname{dif} L)+\frac{W(P ; \operatorname{dif} L) E(L, f)\|P\|}{r}+\frac{E(L, f)}{r} \\
& \leqslant \frac{3 W(P ; \operatorname{dif} L) E(L, f)\|P\|}{r}
\end{aligned}
$$

что и требовалось доказать. 
2.13a. ЗАмЕЧАНИЕ. В правой части неравенства $(2.13 ; 1)$ множитель 3 нельзя заменить меньшим числом. На это указывает следуюший пример.

На $\Delta=[-1,1]$ определим семейство $L \subset M(\Delta)$ равенством $L=\{l=l(x)=c x$ : $c \in \mathbb{R}\}$ и возьмем $P(\cdot)=|\cdot|_{p, \Delta}$ при $p=\left(p_{-}, p_{+}\right)$с $p_{-}(x)=1$ при $x=0, p_{-}(x)=0$ при $x \neq 0, p_{+}(x) \equiv 1$. Тогда для $f=f(x) \equiv 1$ получаем равенства

$$
E(L, f)=1, \quad E(P ; L, f)=1, \quad W(P ; L)=1, \quad W(P ; L(f))=3 .
$$

\section{§4. Устойчивость жесткости и свободы при вариациях веса}

2.14. ТЕОРЕма (об устойчивости жесткости и свободы). $E c \Omega u L \subset M(E)$, $E \subset \mathbb{R}$, знакочувствительные веса $p$ и q ограничень на $E, d=d(p, q)$, $\underline{\sigma}=\underline{\sigma}(p, q, L ; d), m o$

$$
|S(q, L)-S(p, L)| \leqslant \underline{\sigma}:=\min \{\|p\|,\|q\|\} \cdot \omega_{s g}(L, d+0)+d .
$$

Если при этом $\underline{\sigma}<s:=S(p, L)$, то

$$
\frac{W(p, L)}{1+\underline{\sigma} W(p, L)} \leqslant W(q, L) \leqslant \frac{W(p, L)}{1-\underline{\sigma} W(p, L)}=\frac{1}{s-\underline{\sigma}} .
$$

ДокАЗАТЕЛЬСтво. 1. Покажем, что

$$
S(q, L) \leqslant S(p, L)+\|p\| \cdot \omega_{s g}(L, d+0)+d .
$$

Пусть $h>0, v \in L, v \not \equiv 0$,

$$
\begin{gathered}
\frac{|v|_{p, E}}{\|v\|}<S:=S(p, L)+h, \quad l(x):=\frac{v(x)}{\|v\|}, \quad l^{-}:=l^{-}(x)=l(x)^{-}, \\
l^{+}:=l^{+}(x)=l(x)^{+}, \quad y_{-}(x):=\min \left\{\frac{S}{l^{-}},\|p\|\right\} \\
y_{+}(x):=\min \left\{\frac{S}{l^{+}},\|p\|\right\}, \quad x \in E .
\end{gathered}
$$

Тогда $p_{+}(x) \leqslant y_{+}(x) \quad$ (поскольку $\quad l^{+} p_{+}(x) \leqslant|l|_{p, E}<S, \quad$ так $\quad$ что $\quad p_{+}(x) \leqslant$ $\left.\min \left\{S / l^{+},\|p\|\right\}=y_{+}(x)\right)$ и, аналогично, $p_{-}(x) \leqslant y_{-}(x)$.

Определим вес $Y(d ; x)$ равенством

$$
Y(d ; x)=\left(\sup \left\{y_{-}(t):|t-x| \leqslant d\right\}+d, \sup \left\{y_{+}(t):|t-x| \leqslant d\right\}+d\right)
$$

и покажем, что

$$
S(Y(d), L) \leqslant S+\|p\| \omega_{s g}(L, d)+d .
$$

Поскольку $0 \leqslant l^{-} \leqslant\|l\|=1$ и $0 \leqslant l^{+} \leqslant 1$, то для этого достаточно показать, что при каждом $x \in E$ выполняются неравенства

$$
\begin{aligned}
& l^{-} \cdot \sup \left\{y_{-}(t):|t-x| \leqslant d\right\} \leqslant S+\omega_{s g}(L, d)\|p\|, \\
& l^{+} \cdot \sup \left\{y_{+}(t):|t-x| \leqslant d\right\} \leqslant S+\omega_{s g}(L, d)\|p\| .
\end{aligned}
$$


Фиксируем какое-либо $x \in E$. Пусть сначала

$$
\sup \left\{y_{+}(t):|t-x| \leqslant d\right\}<\|p\|
$$

так что при $|t-x| \leqslant d$ имеем

$$
\begin{aligned}
y_{+}(t) & =\frac{S}{l^{+}(t)}, \\
\sup \left\{y_{+}(t):|t-x| \leqslant d\right\} & =\frac{S}{\inf \left\{l^{+}(t):|t-x| \leqslant d\right\}} .
\end{aligned}
$$

Поэтому

$$
\begin{aligned}
l^{+} \cdot & \sup \left\{y_{+}(t):|t-x| \leqslant d\right\} \\
= & \inf \left\{l^{+}(t):|t-x| \leqslant d\right\} \cdot \sup \left\{y_{+}(t):|t-x| \leqslant d\right\} \\
& +\left(l^{+}(x)-\inf \left\{l^{+}(t):|t-x| \leqslant d\right\}\right) \cdot \sup \left\{y_{+}(t):|t-x| \leqslant d\right\} \\
\leqslant & S+\omega_{+}(L, d)\|p\| .
\end{aligned}
$$

В случае

$$
\sup \left\{y_{+}(t):|t-x| \leqslant d\right\}=\|p\|
$$

имеем

$$
\begin{aligned}
\|p\| & =\sup \left\{\min \left\{\frac{S}{l^{+}(t)},\|p\|\right\}:|t-x| \leqslant d\right\} \\
& =\min \left\{\frac{S}{\inf \left\{l^{+}(t):|t-x| \leqslant d\right\}},\|p\|\right\}, \\
\|p\| & \leqslant \frac{S}{\inf \left\{l^{+}(t):|t-x| \leqslant d\right\}},
\end{aligned}
$$

так что $\inf \left\{l^{+}(t):|t-x| \leqslant d\right\} \cdot\|p\| \leqslant S$. Тогда

$$
\begin{aligned}
l^{+} \sup \left\{y_{+}(t):|t-x| \leqslant d\right\} & =l^{+}(x)\|p\| \leqslant\left(\inf \left\{l^{+}(t):|t-x| \leqslant d\right\}+\omega_{+}(L, d)\right)\|p\| \\
& \leqslant S+\omega_{+}(L, d)\|p\| .
\end{aligned}
$$

Аналогично доказываются неравенства $(2.14 ; 6)$ и $(2.14 ; 8)$ с заменой в них знака + знаком - . Итак, неравенства $(2.14 ; 4)$ доказаны. Как уже отмечалось, из них следует $(2.14 ; 3)$.

Так как $p_{-}(x) \leqslant y_{-}(x), p_{+}(x) \leqslant y_{+}(x)$ и $d(p, q)=d$, то из определения веса $Y(d ; x)$ следует, что

$$
q_{-}(x) \leqslant Y_{-}(t ; x), \quad q_{+}(x) \leqslant Y_{+}(t ; x)
$$

(при всех $t>d)$,

$$
S(q, L) \leqslant S(Y(t), L) \leqslant S(p, L)+h+\|p\| \omega_{s g}(L, t)+t,
$$


откуда

$$
S(q, L) \leqslant S(p, L)+\|p\| \omega_{s g}(L, t)+t, \quad S(q, L) \leqslant S(p, L)+\|p\| \omega_{s g}(L, d+0)+d .
$$

2. Докажем теперь неравенство

$$
S(p, L) \leqslant S(q, L)+\|p\| \omega_{s g}(L, d+0)+d
$$

Положим $r_{-}(x)=\min \left\{q_{-}(x),\|p\|\right\}, r_{+}(x)=\min \left\{q_{+}(x),\|p\|\right\}, r=\left(r_{-}, r_{+}\right)$. Очевидно, $d_{1}:=d(p, r) \leqslant d(p, q)=d$. Взяв в рассуждениях п. 1 вместо $p$ вес $r$, а вместо $q$ вес $p$, получим неравенство

$$
S(p, L) \leqslant S(r, L)+\|r\| \omega_{s g}\left(L, d_{1}+0\right)+d_{1} \leqslant S(q, L)+\|p\| \omega_{s g}(L, d+0)+d
$$

3. Из пп. 1, 2 получаем

$$
|S(p, L)-S(q, L)| \leqslant\|p\| \omega_{s g}(L, d+0)+d
$$

Поменяв здесь местами $p$ и $q$, получим еще одно неравенство. Эти два неравенства эквивалентны $(2.14 ; 1)$. Неравенство $(2.14 ; 2)$ при условии $\underline{\sigma}(d)<S(p, L)$ непосредственно следует из $(2.14 ; 1)$ и равенств $S(p, L)=1 / W(p, L)$ и $S(q, L)=1 / W(q, L)$.

Отметим, что условие $\underline{\sigma}(d)<S(p, L)$ нужно лишь для правого из неравенств $(2.14 ; 2)$.

2.14a. ЗАМЕчАнИЕ. Теорема 2.14 и ее доказательство сохраняются при замене $\underline{\sigma}=\underline{\sigma}(p, q, L ; d)$ на $\sigma_{\text {dif }}=\sigma(p, q, \operatorname{dif} L ; d)($ см. п. $\left.2.8,6)\right), S(p, L)-$ на $S(p, \operatorname{dif} L)$ и т.п., так что, в частности, при $\sigma_{\text {dif }}<S(p, \operatorname{dif} L)$ имеем неравенство

$$
W(q, \operatorname{dif} L) \leqslant \frac{1}{S(p, \operatorname{dif} L)-\sigma_{\mathrm{dif}}}, \quad d=d(p, q) .
$$

2.15. Приведем еще два неравенства, вытекающих из теоремы 2.14 и касаюшихся непрерьвной зависимости $W(p, L)$ и $S(p, L)$ от веса $p$. Заметим, что величины вида

$$
r\left(f, f_{0}\right):=\frac{\left|f-f_{0}\right|}{f_{0}}, \quad k\left(f, f_{0}\right):=\frac{\left|\frac{f}{f_{0}}-\frac{f_{0}}{f}\right|}{2},
$$

стоящие в левых частях неравенств в следующей теореме, естественно назвать относительной и обобщенной расстройками величины $f_{0}$ по аналогии с относительной и обобщенной расстройками частоты $f_{0}$, широко используемыми в радиотехнике (считаем $f, f_{0} \in[0, \infty), r(0,0)=k(0,0)=0, r(a, 0)=k(a, 0)=\infty$ при $a>0)$. Очевидно,

$$
\begin{aligned}
& k\left(f, f_{0}\right)=k\left(f_{0}, f\right) \geqslant r\left(f, f_{0}\right) \cdot\left(1-\frac{r\left(f_{0}, f\right)}{2}\right), \\
& r\left(f, f_{0}\right)=k\left(f, f_{0}\right) \cdot 2 f\left(f+f_{0}\right)^{-1} \leqslant 2 k\left(f, f_{0}\right) .
\end{aligned}
$$


2.16. ТЕОРема (о непрерывности расстроек жесткости и свободы). Пусть $L \subset$ $M(E), \quad p$ и - знакочувствительные веса на $E, d=d(p, q), \underline{\sigma}=\underline{\sigma}(p, q, L ; d)$ $($ см n.2.8,4)). Тогда при $\underline{\sigma}<S(p, L)$ имеем

$$
\begin{aligned}
& r\left(W, W_{0}\right) \leqslant \frac{\underline{\sigma} W_{0}}{1-\underline{\sigma} W_{0}}, \\
& k\left(W, W_{0}\right)=k\left(S, S_{0}\right) \leqslant \frac{\underline{\sigma} W_{0}}{1-\underline{\sigma} W_{0}}=\frac{\underline{\sigma}}{S_{0}-\underline{\sigma}},
\end{aligned}
$$

где $W_{0}=W(p, L), \quad W=W(q, L), \quad S_{0}=S(p, L), \quad S=S(q, L)$.

ДоказАтельство. Пусть сначала $W \geqslant W_{0}$. Тогда

$$
k\left(W, W_{0}\right)=\frac{\frac{W}{W_{0}}-\frac{W_{0}}{W}}{2}=\frac{W-W_{0}}{W_{0}} \frac{W+W_{0}}{2 W} \leqslant \frac{W-W_{0}}{W_{0}} .
$$

Из правого неравенства $(2.14 ; 2)$ имеем

$$
\frac{W-W_{0}}{W_{0}} \leqslant \frac{\underline{\sigma} W_{0}}{1-\underline{\sigma} W_{0}}
$$

так что

$$
k\left(W, W_{0}\right) \leqslant \frac{W-W_{0}}{W_{0}} \leqslant \frac{\underline{\sigma} W_{0}}{1-\underline{\sigma} W_{0}} .
$$

Если $W<W_{0}$, то

$$
k\left(W, W_{0}\right)=\frac{W_{0}-W}{W_{0}} \frac{W+W_{0}}{2 W}>\frac{W_{0}-W}{W_{0}} .
$$

Из левого же неравенства $(2.14 ; 2)$ имеем $W_{0} / W \leqslant 1+\underline{\sigma} W_{0}$, откуда $W / W_{0} \geqslant 1 /(1+$ $\left.\underline{\sigma} W_{0}\right)$. Вычитая это неравенство из предыдущего, получаем

$$
\left(\frac{W_{0}}{W}-\frac{W}{W_{0}}\right) \leqslant 2 \underline{\sigma} W_{0} \frac{1+\frac{\underline{\sigma} W_{0}}{2}}{1+\underline{\sigma} W_{0}}<2 \underline{\sigma} W_{0}
$$

так что $\left(W_{0}-W\right) / W_{0}<k\left(W, W_{0}\right)<\underline{\sigma} W_{0}$. Доказательство закончено.

Следуюшее утверждение касается зависимости жесткости и свободы системы $(p, L)$ от множества $A$, на котором берется " $p$-норма".

2.17. Теорема. Пусть $A$ и $B$ - подмножества множества $E \subset \mathbb{R}, h-$ хаусдорфово расстояние между $A$ u $B, \quad L \subset M(E), \quad p=\left(p_{-}, p_{+}\right)$- знакочувствительный вес на $E, \omega(p, t)$ - его модуль непрерывности на $E$, $z=z(h):=\omega(p, h+0)+\|p\| \omega(L, h+0)$. Тогда если при $F \subset E$ положить $P_{F}(\cdot):=|\cdot|_{p, F}, \quad S_{F}:=S\left(P_{F} ; L\right)$ u $W_{F}:=W\left(P_{F} ; L\right)$, mo

$$
\begin{gathered}
\left|S_{A}-S_{B}\right| \leqslant\left\|P_{A}-P_{B}\right\|_{L} \leqslant z, \\
\frac{W_{A}}{1+z W_{A}} \leqslant W_{B} \leqslant \frac{W_{A}}{1-z W_{A}}, \quad z<S_{A}=\frac{1}{W_{A}} .
\end{gathered}
$$


ДоКАЗАТЕЛЬСТво. Пусть для определенности $S\left(P_{A} ; L\right) \geqslant S\left(P_{B} ; L\right)$ и $l_{k}$ - такая последовательность функции из $L$, что при $k \rightarrow 0$ имеем

$$
\lim \frac{P_{B}\left(l_{k}\right)}{\left\|l_{k}\right\|}=\inf \left\{\frac{P_{B}(l)}{\|l\|}: l \in L\right\}=S\left(P_{B} ; L\right) .
$$

Тогда для любого $\varepsilon>0$ при достаточно больших $k$ имеем

$$
S\left(P_{A} ; L\right)-S\left(P_{B} ; L\right)-\varepsilon \leqslant \frac{P_{A}\left(l_{k}\right)}{\left\|l_{k}\right\|}-\frac{P_{B}\left(l_{k}\right)}{\left\|l_{k}\right\|} \leqslant\left\|P_{A}-P_{B}\right\|_{L},
$$

откуда получаем левое из неравенств $(2.17 ; 1)$. Считая по-прежнему $\varepsilon>0$, возьмем $l \in L$. По определению $h$ для каждого $x \in A$ найдется $t \in B$, для которого $|x-t|<h+\varepsilon$. Поэтому имеем неравенства

$$
\begin{aligned}
l^{+}(x) p_{+}(x)= & (l(t)+l(x)-l(t))^{+} p_{+}(x) \leqslant l^{+}(t) p_{+}(t)+l^{+}(t)\left(p_{+}(x)-p_{+}(t)\right) \\
& +(l(x)-l(t))^{+} p_{+}(x) \leqslant P_{B}(l)+\|l\|_{B} \omega(p, h+\varepsilon)+\omega(l, h+\varepsilon)\|p\|_{A},
\end{aligned}
$$

а также аналогичные неравенства с заменой индекса + на - . Так как всегда хотя бы одно из чисел $a^{+}, a^{-}$равно нулю, то отсюда

$$
\begin{gathered}
|(l, p)(x)| \leqslant P_{B}(l)+\|l\|_{E}(\omega(p, h+0)+\omega(L, h+0)\|p\|), \quad x \in A \\
P_{A}(l) \leqslant P_{B}(l)+\|l\|_{E} z .
\end{gathered}
$$

Аналогично, $P_{B}(l) \leqslant P_{A}(l)+\|l\|_{E} z$, что вместе с $(2.17 ; 4)$ дает правое из неравенств $(2.17 ; 1)$. Неравенство же $(2.17 ; 2)$ при условии $z<S_{A}$ эквивалентно неравенству $\left|S_{A}-S_{B}\right| \leqslant z$.

\section{Глава 3. Вопросы существования и единственности элемента наилучшего приближения}

Вопрос о сушествовании элемента наилучшего приближения в случае знакочувствительных аппроксимаций является гораздо более сложным, чем можно было предполагать a priori. Например, на отрезке $\Delta=[0,1]$ имеются ограниченный и полунепрерьвньй сверху вес $p=\left(p_{-}, p_{+}\right)$, чебьшевское подпространство $L$ и непрерывная функция $f$, для которых элемент $l(p, L, f)$ не существует. Действительно, пусть $p_{-}(x) \equiv 1 \forall x \in[0,1], p_{+}(0)=1, p_{+}(x)=0 \forall x \in(0,1], L$ - семейство всех линейных функций $l(x)=a x+b, f(x)=x^{1 / 2}$. Тогда $E(p, L, f)=0$, так как

$$
\left|a x-x^{1 / 2}\right|_{p, \Delta}=\max \left\{x^{1 / 2}-a x: x \in[0,1]\right\} \rightarrow 0 \quad \text { при } \quad a \rightarrow+\infty .
$$

Поэтому для элемента наилучшего приближения $l(p, L, f)$, если он сушествует, имеем равенство $l(p, L, f ; 0)=0$, так что $l(p, L, f ; x) \equiv a x$ при некотором $a=$ const. Так как $\left|a x-x^{1 / 2}\right|_{p, \Delta}>0 \forall a$, то $l(p, L, f)$ не сушествует.

В случае ограниченного веса $p$ и чебьшевского подпространства $L$ на отрезке $\Delta$ из условия $E(p, L, f)>0$ следует существование и единственность $l(p, L, f)$ (см. ниже теорему 3.8). Если же отказаться от требования чебышевости $L$ на отрезке $\Delta$, сохранив требование его чебьшевости на незамкнутом промежутке $\Delta$, то 
элемент наилучшего приближения в $L$ для некоторых функций $f \in C(\Delta)$ может не существовать. Например, если $\Delta=[0,1], p_{-}(x) \equiv 1$ и $p_{+}(x) \equiv 0, L$ состоит из всех функций вида $l(x)=a x, a \in \mathbb{R}, f(x)=h+x^{1 / 2}, h>0$, то $E(p, L, f)=h$, но $l(p, L, f)$ не существует, так как $|a x-f(x)|_{p, \Delta}>h$ при любом $a$.

По теореме А. Хаара (см., например, [14]) для того, чтобы любая функция $f \in$ $C(\Delta=[a, b])$ имела единственный полином наилучшего приближения $l=$ $c_{1} \varphi_{1}+\cdots+c_{n} \varphi_{n}$ в $C(\Delta)$ по заданной линейно независимой системе $\left\{\varphi_{k}\right\}_{k=1}^{n} \subset$ $C(\Delta)$, необходимо и достаточно, чтобы эта система была чебышевской на $\Delta$. Это верно и для аппроксимаций с непрерьвньм и положительньг весом $p=\left(p_{-}, p_{+}\right)$ на $\Delta[12$, с. 491$]$. В случае же произвольного знакочувствительного веса (вообще говоря, с точками вырожденности и полувырожденности) дело обстоит гораздо сложнее, хотя и в этом случае чебышевость приближаюшего подпространства (но уже на подмножествах отрезка $\Delta$ ) в вопросах единственности является критериальным свойством системы $\left\{\varphi_{k}\right\}_{k=1}^{n}$ (см. [15]-[17]). При этом, в частности, возникает задача описания семейства всех весов $p$, для которых у любой функции $f \in C(\Delta)$ ее элемент наилучшего приближения чебьшевским подпространством $L$ является единственным.

Отметим, что в случае $E(p, L, f)>0$ при непрерывности $f$, чебышевости $L$ и ограниченности веса $p=\left(p_{-}, p_{+}\right)$элемент наилучшего приближения $l(p, L, f)$ существует и единствен (теорема 3.8), и если при этом вес $p$ полунепрерывен сверху, то, как в классическом случае чебьшевских аппроксимаций, справедливы также и естественные обобшения теоремы Чебьшева об альтернансе (теорема 3.6) и теоремы Валле-Пуссена (теорема 3.7). Поэтому столь серьезное внимание мы уделяем вопросам сушествования и единственности $l(p, L, f)$ в особом случае $E(p, L, f)=0$, тривиальном в классических аппроксимациях. Ряд результатов доказьвается для общего случая аппроксимаций в нормированных пространствах относительно сублинейного функционала.

\section{$\S 1$. Теоремы существования элемента наилучшего приближения}

Сначала сделаем простые замечания общего характера об условиях существования элемента наилучшего приближения.

3.0. а) Если $L$ - компакт в линейном метрическом пространстве $\mathscr{L}, P$ - полунепрерывньй снизу неотрицательньй функционал на $\mathscr{L}$, то каждый элемент $f \in \mathscr{L}$ имеет в $L$ хотя бы один элемент наилучшего приближения.

б) Пусть $L$ - такое замкнутое множество в линейном нормированном пространстве $\mathscr{L}$, что его пересечение с любым замкнутым шаром в $\mathscr{L}$ является компактом, $f \in \mathscr{L}$, а $P$ - такой полунепрерывный снизу неотрицательный функционал на $\mathscr{L}$ с ядром $N(P)$, что $P(l) \rightarrow \infty$ при $\|l\| \rightarrow \infty, l \in L(f) \backslash N(P)$ (последнее вьполняется, в частности, при $W(P ; L(f))<\infty)$. Тогда у $f$ в $L$ имеется по меньшей мере один элемент наилучшего приближения.

3.1. ТеОрема. Пусть $P$ - произвольный полунепрерывный снизу неотрицательный функционал на линейном нормированном пространстве $\mathscr{L}$,

$$
\Psi(P, t):=\sup \left\{\frac{P(l)}{P(t l)}: l \in \mathscr{L},\|l\|=1,0<P(t l)<\infty\right\} \rightarrow 0 \quad \text { npu } \quad t \rightarrow \infty,
$$


$L$ - такое замкнутое множество в $\mathscr{L}$, что его чентральная проекция $L_{0}:=$ $\{l /\|l\|: l \in L, l \neq 0\}$ на единичную сферу в пространстве $\mathscr{L}$ является предкомпактом, $и W\left(P ; \bar{L}_{0}\right)<\infty$. Тогда каждый әлемент $f \in \mathscr{L}$ имеет в L хотя бы один әлемент наилучшего приближения $l(P ; L, f)$.

ДоказАтельство. Пусть $f \in \mathscr{L}$. Покажем, что $P(g) \rightarrow \infty$ при $g \in$ $L(f) \backslash N(P), \quad\|g\| \rightarrow \infty$. Возьмем последовательность $\left\{g_{k}\right\} \subset L(f) \backslash N(P)$ с $P\left(g_{k}\right)<\infty,\left\|g_{k}\right\| \rightarrow \infty$. Тогда $g_{k}=l_{k}-f$, где $l_{k} \in L$, и (см. $\left.(3.1 ; 1)\right)$

$$
\frac{P\left(\frac{g_{k}}{\left\|g_{k}\right\|}\right)}{P\left(g_{k}\right)} \rightarrow 0, \quad k \rightarrow \infty .
$$

Пусть $h$ - какая-либо предельная точка последовательности $l_{k} /\left\|l_{k}\right\|\left(h \in \bar{L}_{0}\right)$. Так как $S\left(P ; \bar{L}_{0}\right)>0$, то $P(h)>0$. А так как $h$ является предельной точкой и для последовательности $\left\{g_{k} /\left\|g_{k}\right\|\right\}$, то

$$
\underline{\lim } P\left(\frac{g_{k}}{\left\|g_{k}\right\|}\right) \geqslant P(h)>0
$$

Отсюда и из $(3.1 ; 2)$ имеем $P\left(g_{k}\right) \rightarrow \infty, k \rightarrow \infty$.

Итак, $P(g) \rightarrow \infty$ при $\|g\| \rightarrow \infty$ и $g \in L(f) \backslash N(P)$. Заметим еще, что из компактности $L_{0}$ вытекает компактность пересечения $L$ с любым шаром из $\mathscr{L}$. Отсюда и из п. 3.0, б) следует доказываемое утверждение.

3.1а. СлЕДСТвИЕ. Если $P$ - произвольный неотрицательный и полунепрерывный снизу положительно однородный функционал на линейном нормированном пространстве $\mathscr{L}, L$ - конечномерное подпространство в $\mathscr{L}$, $W(P ; L)<\infty$, то калдыи әлемент $f \in \mathscr{L}$ имеет в $L$ хотя бы один әлемент наилучшего приближения $l(P ; L, f)$.

3.1б. СлЕДСтвИЕ. Каковы бы ни были множество $E$, знакочувствительный вес $p$ на $E$ и конечномерное подпространство $L \subset M(E)$, при $W(p, L)<\infty$ каждая функция $f \in M(E)$ имеет в $L$ хотя бы один элемент наилучшего приближения $l(p, L, f)$.

Условие $W(p, L)<\infty$ здесь нельзя опустить даже в случае $E=[a, b]$, ограниченного знакочувствительного веса $p$, чебышевского подпространства $L \subset C([a, b])$ и функции $f \in C([a, b])$. На это указывает приводимая ниже теорема 3.5 .

3.2. ТеОРема. Пусть $P$ - полунепрерывный снизу строго выпуклый сублинейный функиионал на линейном нормированном пространстве $\mathscr{L}, L-$ конечномерное линейное подпространство в $\mathscr{L}, W(P ; L)=\infty, f \in \mathscr{L}$. При этих условиях $l(P ; L, f)$ существует тогда и только тогда, когда $f$ представима в виде $f=l-g$, где $l$ принадлежит $L$, а $g$ - ядру $N(P)$ функционала $P$.

ДокаЗАтеЛЬСтво. Необходимость. Пусть $l(P ; L, f)=: l$ сушествует. Предположим, что $l-f \notin N(P)$, т.е. $P(l-f)=E(P ; L, f)>0$. Поскольку $W(P ; L)=\infty$, то существует такой элемент $v \in L$, что $\|v\|=1, P(v)=0$ (см. теорему 2.1). Очевидно, не может быть $l-f=t v$ для какого-либо $t=$ const $\geqslant 0$, 
так что $l-f$ и $v$ неколлинеарны. Отсюда и из строгой выпуклости $P$ получаем противоречие:

$$
E(P ; L, f) \leqslant P(l+v-f)<P(l-f)+P(v)=P(l-f)=E(P ; L, f) .
$$

Поэтому $l-f \in N(P)$.

Достаточность. Пусть $f=l-g$, где $l \in L, g \in N(P)$. Тогда

$$
0 \leqslant E(P ; l, f) \leqslant P(l-f)=P(g)=0,
$$

так что $l=l(P ; L, f)$.

3.2а. СлЕДСТвИЕ. Если при условиях и обозначениях теоремы 3.2 имеем $E(P ; L, f)>0$, то әлемент $l(P ; L, f)$ не существует (иначе было бы $0<$ $E(P ; L, f) \leqslant P(l-f)=P(g)=0)$.

3.2б. ЗАмечаниЕ. При условиях теоремы 3.2 равенство $E(P ; L, f)=0$ необходимо (по $3.2 \mathrm{a})$, но не достаточно для существования $l(P ; L, f)$, на что указывает следующий пример. Пусть $\mathscr{L}=\mathbb{R}^{2}$ - пространство двумерных векторов $f=(x, y), P(f)=\left(x^{2}+y^{2}\right)^{1 / 2}-x$. Тогда $P$ - положительный строго выпуклый непрерывный сублинейный функционал с ядром $N=\{(x, 0): x \geqslant 0\}$. При $L=\mathbb{R}=\{(x, 0):-\infty<x<\infty\}$ имеем $N \subset L$, так что $W(P ; L)=\infty$ (см. $(2.0 ; 2))$. При этом $E(P ; L, f)=0$ для любого $f=(u, v)$ (для $l=(x, 0)$ имеем $P(l-f)=\left((x-u)^{2}+v^{2}\right)^{1 / 2}-(x-u) \rightarrow 0$ при $\left.x \rightarrow \infty\right)$, в то время как $P(l-f)>0$ для любых $l \in L$ и $f \notin L$. Итак, каков бы ни был элемент $f \notin L$, у него нет элемента наилучшего приближения $l(P ; L, f)$, хотя $E(P ; L, f)=0$.

Далее в этом параграфе мы занимаемся лишь аппроксимациями со знакочувствительным весом элементами чебышевских подпространств. Нам понадобится следующее вспомогательное утверждение.

3.3. Лемма. Пусть $L$ - чебышевское подпространство на отрезке $\Delta$, $p$ - вес на $\Delta, \quad W(p, L)=\infty, \quad m$ - некоторая функчия из $L \quad(m \neq \equiv 0)$, для которой $|m|_{p, \Delta}=0$. Тогда если вес $p$ ограничен в некоторой окрестности $U$ множества нулей функиии $m$, то $E(p, L, f)=0 \quad \forall f \in C(\Delta)$.

ДокаЗАтеЛЬСтво. Пусть $l \in L, \quad l(x)$ интерполирует $f(x)$ в нулях $m$, $t=$ const $>0$. Через $\Delta(t)$ обозначим множество всех $x \in \Delta$, в которых $\operatorname{tm}(x)-$ $(f(x)-l(x))$ отлично от нуля и имеет тот же знак, что и $m(x)$. Очевидно, множество $E(t):=\Delta \backslash \Delta(t)$ попадает в $U$ при достаточно больших $t$. Так как при этом вес $p$ ограничен на $U$ и $\|t m-g\|_{E(t)} \rightarrow 0$ при $t \rightarrow \infty$, то отсюда $|t m-(f-l)|_{p, \Delta} \rightarrow 0$ при $t \rightarrow \infty$, так что $E(p, L, f)=0$.

3.3a. ЗАмЕчАниЕ. В условиях доказанного утверждения нельзя заменить ограниченность веса $p$ в $U$ его ограниченностью (конечностью) на множестве нулей $m$. Например, если $\Delta=[-1,1], L=\{l: l(x)=a x+b: a, b \in \mathbb{R}\}, p_{-}(x)=0$ при $x \leqslant 0, p_{-}(x)=x^{-1 / 2}$ при $x>0, p_{+}(x)=p_{-}(-x), f(x)=|x|^{1 / 2} \cdot \operatorname{sign} x$, то $|a x|_{p, \Delta}=0$ при $a<0, W(p, L)=\infty$, но $E(p, L, f)=1$ (и любой полином $l(x)=a x$ c $a \geqslant 0$ является полиномом наилучшего приближения).

Непосредственно из утверждений 3.3 и 3.1 а вытекает 
3.4. Теорема. Пусть $L$ - чебышевское подпространство на отрезке $\Delta$, $p$ - ограниченный знакочувствительный вес на $\Delta u E(p, L, f)>0$ для некоторой функиии $f \in C(\Delta)$. Тогда $W(p, L)<\infty$ и каждая функиия из $C(\Delta)$ имеет в L хотя бы один әлемент наилучшего приближения.

Следуюшая теорема является основной в этом параграфе.

3.5. Теорема. Пусть $L-$ чебышевское подпространство на отрезке $\Delta$, $p=\left(p_{-}, p_{+}\right)$- ограниченный вес на $\Delta$. Тогда для наличия у каждой функции $f \in C(\Delta)$ хотя бь одного әлемента наилучшего приближения $l(p, L, f)$ необходимо и достаточно выполнения одного из следующих условий:

a) $W(p, L)<\infty$;

б) $W(p, L)=\infty$ икаждая точка множества $\operatorname{supp}\left(p_{-}\right) \cap \operatorname{supp}\left(p_{+}\right)$является изолированной точкой множества $\operatorname{supp}\left(p_{-}\right) \cup \operatorname{supp}\left(p_{+}\right)$.

ДокАЗАТЕльСТво. Напоминаем (см. п. $2.7 \mathrm{a} ; 0)$, что

$$
\operatorname{supp}(p):=\operatorname{supp}\left(p_{-}\right) \cup \operatorname{supp}\left(p_{+}\right), \quad \Pi(p):=\operatorname{supp}\left(p_{-}\right) \cap \operatorname{supp}\left(p_{+}\right) .
$$

3.5a. В случае а) сушествование $l(p, L, f)$ для каждой $f \in C(\Delta)$ следует из свойств функционала $P(\cdot)=|\cdot|_{p, \Delta}($ см. п. 0.1) и из 3.1а. Если для каждой $f \in C(\Delta)$ существует $l(p, L, f)$, то имеем либо уже рассмотренньй случай $W(p, L)<\infty$, либо случай $W(p, L)=\infty$.

3.5б. Рассматриваем случай $W(p, L)=\infty$.

Необходимость. 3.5б;1. Пусть $l(p, L, f)$ существует для любой функции $f \in C$ $(\Delta=[a, b])$. Если П $(p)$ пусто, то все доказано. Пусть П $(p)$ не пусто, $t \in \Pi(p)$. Допуская существование точки $t \in \Pi(p)$, предельной для $\operatorname{supp}(p)$, для определенности предположим, что точка $t$ является предельной справа для $\operatorname{supp}\left(p_{-}\right)$(тогда $t<b)$. Положим (см. п. 2.8) $f(x)=(\omega(L, x-t))^{1 / 2}$ при $x \geqslant t, f(x)=0$ при $x<t$.

$3.5 б ; 2$. Покажем, что $l(p, L, f ; t)=0$. Так как $W(p, L)=\infty$, то $E(p, L, f)=0$ (см. пा. $3.3,2.1,0.1)$. Если бы для $l=l(p, L, f)$ было $l(t) \neq 0=f(t)$, то из непрерывности $f$ и $l$ следовало бы, что в некоторой окрестности точки $t$ имеем либо $l(x)-f(x)<0$, либо $l(x)-f(x)>0$. Так как $t \in \Pi(p)$, то в любой окрестности точки $t$ функция $(l-f, p)(x)$ не тождественна нулю, откуда $E(p, L, f)>0$. А это не так.

$3.5 б ; 3$. Поскольку $l(t)=0$, то для точек $x>t$, близких к $t$, имеем из определения $\omega(L, r)($ см. п.2.8, 2)) неравенства

$$
l(x) \leqslant\|l\| \cdot \omega(L, x-t)<\omega(L, x-t)^{1 / 2}=f(x), \quad l(x)<f(x) .
$$

В тех $x$, в которых $p_{-}(x)>0$ (напомним, что точка $t$ предельная справа для $\left.\operatorname{supp}\left(p_{-}\right)\right)$, имеем также неравенство $(l-f, p)(x)<0$. А это противоречит равенству $E(p, L, f)=0$. Необходимость условий теоремы в случае $W(p, L)=\infty$ доказана.

Достаточность. Пусть каждая точка множества П $(p)$ является изолированной точкой множества $\operatorname{supp}(p)$ (см. $(3.5 ; 0))$.

$3.5 б ; 4$. Заметим прежде всего, что при рассматриваемом условии $W(p, L)=\infty$ множество П $(p)$ имеет не более $n-1$ точек, $n=\operatorname{dim} L$, так как в противном случае 
(см. п. 2.7а) было бы $W(p, L)<\infty$. Заметим еше, что при условии $W(p, L)=\infty$ точки множеств $\operatorname{supp}\left(p_{-}\right)$и $\operatorname{supp}\left(p_{+}\right)$не могут и сильно перемежаться (см. случай 2.5, a) теоремы 2.7$)$. Помня об этом, рассмотрим два случая.

3.5б;5. Пусть $\operatorname{supp}\left(p_{-}\right)=\operatorname{supp}\left(p_{+}\right)=\Pi(p)=\operatorname{supp}(p)$. Тогда любой элемент $l \in L$, интерполирующий $f(x)$ в точках множества П $(p)$, является элементом наилучшего приближения для $f$ и $E(p, L, f)=0$.

3.5б;6. Пусть теперь $\operatorname{supp}\left(p_{-}\right) \neq \operatorname{supp}\left(p_{+}\right)$. Так как точки $x \in \Pi(p)$ являются изолированными точками множества $\operatorname{supp}(p)$ и П $(p)$ состоит из конечного числа точек, то на $\Delta=[a, b]$ найдется некоторое конечное число $k$ таких отрезков $D_{j}=$ $\left[c_{j}, d_{j}\right], j=1, \ldots, k$, что $a \leqslant c_{1}<d_{1}<c_{2}<d_{2}<\cdots<c_{k}<d_{k} \leqslant b$, и при этом выполняются условия:

а) объединение всех этих отрезков покрьвает множество $\operatorname{supp}(p) \backslash \Pi(p)$ и не пересекается с $\Pi(p)$;

б) каждый из отрезков $D_{j}$ либо содержит точки множества $\operatorname{supp}\left(p_{-}\right)$, но не содержит точек из $\operatorname{supp}\left(p_{+}\right)$(такой отрезок $D_{j}$ будем называть $(-)$-отрезком), либо $D_{j}$ содержит точки из $\operatorname{supp}\left(p_{+}\right)$, но не содержит точек из $\operatorname{supp}\left(p_{-}\right)$(тогда $D_{j}$ назовем $(+)$-отрезком); при этом если между соседними отрезками $D_{j}$ и $D_{j+1}$ нет точек из П $(p)$, то эти отрезки имеют “разные знаки”. Середины $\left(d_{j}+c_{j+1}\right) / 2$ интервалов $\left(d_{j}, c_{j+1}\right)$, на которых нет точек из $\Pi(p)$, соберем в конечное множество $Q$ (оно может быть и пустым).

3.5б;7. Приступаем к построению функции $r \in L$, равной нулю во всех точках $x_{q} \in \Pi(p) \cup Q$, положительной на $(-)$-отрезках $D_{j}$, отрицательной на $(+)$-отрезках $D_{j}$ и при этом имеющей на $\Delta$ минимально возможное общее число нулей с учетом их четности $n\left(x_{q}\right)$ (см. начало $\S 2$ гл. 2). Все точки $x_{q} \in Q$ будут нечетными нулями для $r$. Если отрезки $D_{j}$ и $D_{j+1}$ имеют один и тот же знак, а число точек множества $\Pi_{j}:=\Pi(p) \cap\left(d_{j}, c_{j+1}\right)$ четно, то все точки $x_{q} \in \Pi_{j}$ также будут нечетными нулями для $r$. Нечетными нулями будут все точки $x_{q} \in \Pi_{j}$, расположенные между отрезками $D_{j}$ и $D_{j+1}$ разных знаков, если число этих точек нечетно. Нечетными нулями будут также и все точки $x_{q} \in \Pi(p)$, лежашие левее $D_{1}$ и правее $D_{k}$ (если такие точки имеются). Если отрезки $D_{j}$ и $D_{j+1}$ имеют один и тот же знак, а множество $\Pi_{j}$ состоит из нечетного числа точек, или же отрезки $D_{j}$ и $D_{j+1}$ имеют разные знаки, а $\Pi_{j}$ состоит из четного числа точек, то ровно одна из точек множества $\Pi_{j}$ (ее можно выбрать произвольно) будет четным нулем, остальные его точки будут нечетными нулями.

3.5б;8. Прежде всего заметим, что любая функция $r \in L$, равная нулю на $\Pi(p) \cup Q$, положительная на $(-)$-отрезках $D_{j}$ и отрицательная на $(+)$-отрезках $D_{j}$, не может иметь общее число нулей (с учетом их четности) меньше числа $m:=$ $\sum n\left(x_{q}\right)$, где суммирование ведется по всем точкам $x_{q} \in \Pi(p) \cup Q$.

3.5б;9. Покажем, что на $\Delta$ имеются точки $a_{1}<a_{2}<\cdots<a_{m+1}$, в которых $p_{-}(x)$ и $p_{+}(x)$ попеременно положительны. Для этого на каждом $(-)$-отрезке и $(+)$-отрезке возьмем по одной точке, в которой соответственно функция $p_{-}(x)$ или $p_{+}(x)$ положительна. Множество всех этих точек обозначим через $D$.

В каждом из тех непустых множеств $\Pi_{j}$, которые состоят лишь из "нечетных нулей" $x_{q}$, удалим какую-нибудь одну точку. Через $A$ обозначим множество, в которое превратиться П $(p) \cup D$ после этой операции, а через $v$ - число его точек. Занумеруем эти точки в порядке их возрастания в конечную последователь- 
ность $a_{1}<a_{2}<\cdots<a_{v}$. В этих точках функции $p_{-}$и $p_{+}$попеременно положительны, так как между отрезками $D_{j}$ и $D_{j+1}$ разных знаков расположено четное число точек множества $A$, а между отрезками одного знака - нечетное их число (см. выше). При этом, как нетрудно видеть, число отрезков $\left[a_{s}, a_{s+1}\right]$, лежаших на $\left[c_{j}, d_{j+1}\right]=\left[c_{j}, d_{j}\right] \cup\left(d_{j}, c_{j+1}\right) \cup\left[c_{j+1}, d_{j+1}\right]$, равно общему числу "нулей" $x_{q} \in \Pi(p) \cup Q$ (с учетом их четностей $\left.n\left(x_{q}\right)\right)$, попавших на интервал $\left(d_{j}, c_{j+1}\right)$. Аналогично, число отрезков $\left[a_{s}, a_{s+1}\right]$, лежащих на $\left[a, d_{1}\right]$, равно числу "нулей" $x_{q}$ (все они нечетные), попавших на $\left[a, c_{1}\right)$, а число отрезков $\left[a_{s}, a_{s+1}\right]$, лежащих на $\left[c_{k}, b\right]$, равно числу "нулей" $x_{q}$ (они также нечетные), попавших на $\left(d_{k}, b\right]$. Из всего этого следует, что общее число отрезков $\left[a_{s}, a_{s+1}\right], s=1, \ldots, v-1$, равно $m$. Таким образом, $v=m+1$, что и требовалось показать.

3.5б;10. Так как $W(p, L)=\infty$, то по теореме 2.7 имеем неравенства $v=$ $m+1 \leqslant n, m \leqslant n-1$. Поэтому (по лемме 2.4 ) найдется функция $R \in L$, имеющая в каждой точке $x_{q}$ нуль указанной вьше четности $n\left(x_{q}\right)$, причем можно считать, что $R$ не имеет на $\Delta$ других нулей, кроме точек $x_{q}$, за одним возможным исключением: когда разность $n-1-m$ нечетна и при этом ровно один конец отрезка $\Delta$ принадлежит П $(p)$. В этом исключительном случае существует функция $R \in L$ с нулями нужных четностей во всех точках $x_{q}$ и с еще одним нечетным нулем, который можно выбрать как угодно близко к упомянутому концу отрезка $\Delta$. Это следует из леммы 2.4. Итак, в любом случае либо $R(x)$, либо $-R(x)$ обладает свойствами искомой функции $r(x)$, о которой шла речь в п. 3.5б; 7 .

$3.5 б ; 11$. Пусть $f \in C(\Delta)$. Через $s(x)$ обозначим элемент из $L$, интерполирующий $f(x)$ в точках П $(p)$ (этих точек не более $n-1$ ). Из свойств элемента $r(x)$ вытекает, что $\operatorname{tr}(x)+s(x)-f(x)=0$ на П $(p)$ при любом $t$, и при достаточно больших $t>0$ имеем неравенство

$$
\min \left\{|\operatorname{tr}(x)|: x \in \bigcup_{j=1}^{k} D_{j}\right\}>\|s-f\|_{\Delta},
$$

и при этом на каждом $(+)$-отрезке $D_{j}$ функция $\operatorname{tr}(x)+s(x)-f(x)$ отрицательна, а на каждом (-)-отрезке $D_{j}$ - положительна. Отсюда $(t r+s-f, p)(x) \equiv 0$, так что $\operatorname{tr}(x)+s(x)$ является при этих $t$ элементом наилучшего приближения для $f \mathrm{c}$ весом $p$. Теорема доказана.

\section{§2. Чебышевская теория}

Следуюшая теорема обобшает теорему П. Л. Чебьшева об альтернансе на аппроксимации со знакочувствительным весом. Отметим, что обобщение теоремы П. Л. Чебышева об альтернансе на случай равномерного приближения полиномами по чебышевской системе (в наших обозначениях при $p \equiv(1,1))$ дано С. Н. Бернштейном (см. [18]), которым также введен и сам термин "чебышевская система".

Сначала дадим определение $p$-альтернанса.

ОПреДЕЛЕНИЕ. Пусть $E \subset \mathbb{R}, p$ - знакочувствительный вес на $E, f, g \in M(E)$. Назовем семейство точек $x_{1}<x_{2}<\cdots<x_{n}$ из $E n$-точечным $p$-альтернансом пары $f, g$ (на $E$ ), если с возрастанием целого $k$ от 1 до $n$ величина $(f-g, p)\left(x_{k}\right)$ с каждым шагом меняет знак, оставаясь по абсолютной величине равной $|f-g|_{p, E}$. 
Если $E=\Delta$ - промежуток, $L-n$-мерное чебышевское подпространство на $\Delta$, $l \in L, f \in C(\Delta)$, то $(n+1)$-точечный $p$-альтернанс пары $l, f$ (если он существует) будем называть ее чебышевским р-альтернансом.

3.6. Теорема (о чебьшевском $p$-альтернансе). Пусть $L-n$-мерное чебышевское подпространство на отрезке $\Delta(n \geqslant 1), p=\left(p_{-}, p_{+}\right)$- конечный $и$ полунепрерывный сверху вес на $\Delta, f \in C(\Delta), E(p, L, f)>0$. Тогда для того чтобы функиия $l \in L$ была әлементом наилучшего приближения в $L$ для $f c$ весом р, необходимо и достаточно наличие у пары $l, f$ на $\Delta(n+1)$-точечного р-альтернанса.

Доказательство этой теоремы аналогично доказательству теоремы П. Л. Чебышева [19] (см. в [12, теорему 5.3 гл. IX]).

Аналогично доказьвается и следующее обобщение на знакочувствительный вес теоремы С. Н. Бернштейна [18] (в свою очередь обобшаюшей теорему Валле-Пуссена [20] на чебьшевские системы).

3.7. ТЕОремА. Если $L-n$-мерное чебышевское подпространство на промежутке $\Delta, \quad l \in L, \quad x_{1}<x_{2}<\cdots<x_{n+1}$ - некоторый набор точек из $\Delta$, $s=\min \left\{\left|(l-f, p)\left(x_{k}\right)\right|: k=1, \ldots, n+1\right\}>0, \operatorname{sign}(l-f, p)\left(x_{k}\right)=-\operatorname{sign}(l-$ $f, p)\left(x_{k+1}\right), k=1, \ldots, n, \operatorname{mo} E(p, L, f) \geqslant s$.

Заметим, что для справедливости теоремы 3.6 о чебышевском $p$-альтернансе в части необходимости ее условий весьма существенно то, что рассматриваемый промежуток $\Delta$ является отрезком. На это, в частности, указывает следуюший пример. Пусть $\Delta=(0,1], p(x) \equiv(1,1), f(x)=\left(1-e^{-1 / x}\right) \sin (1 / x), L_{n}$ - семейство всех действительнозначных алгебраических полиномов степени $\leqslant n$. Тогда при каждом $n \geqslant 1$ имеем $E\left(p, L_{n}, f\right)=1$, и алгебраический полином наилучшего приближения $P\left(p, L_{n}, f ; x\right)$ степени $\leqslant n$ для $f$ тождествен нулю (действительно, в противном случае $P^{(k)}\left(p, L_{n}, f ; 0\right) \neq 0, P^{(r)}\left(p, L_{n}, f ; 0\right)=0$ при некотором неотрицательном $k \leqslant n$ и $r<k$ и на некотором интервале $(0, h)$ будет

$$
\left|P\left(p, L_{n}, f ; x\right)\right|>C x^{k}, \quad C-\text { const }>0
$$

так что разность $P\left(p, L_{n}, f ; x\right)-f(x)$ в любой близости от нуля справа при некоторых $x$ принимает значения, меньшие -1 или большие +1$)$. Ясно, что в этом случае нет ни одной точки альтернанса. Этот пример нетрудно обобщить на произвольные промежутки $\Delta$, не являюшиеся отрезками, и на произвольные чебышевские подпространства (особенно просто - в случае непрерывной продолжимости функций из $L$ на замыкание промежутка $\Delta$ ).

В части же достаточности условий теоремы 3.6 , как нетрудно видеть, от $\Delta$ можно требовать лишь того, чтобы это был промежуток $\langle a, b\rangle$.

Следуюшее утверждение обобщает теорему П. Л. Чебьшева о единственности алгебраического полинома наилучшего равномерного приближения для $f \in$ $C([a, b])$ (на равномерные приближения полиномами по произвольной чебьшевской системе теорему П. Л. Чебышева и ее доказательство распространил А. Хаар; см., например, [14]). 
3.8. Теорема. Пусть $L$ - чебышевское подпространство на отрезке $\Delta$, вес $p=\left(p_{-}, p_{+}\right)$ограничен на $\Delta$. Тогда каждая функиия $f \in C(\Delta)$, для которой $E(p, L, f)>0$, имеет әлемент наилучшего приближения $l(p, L, f)$, притом единственный.

ДокАЗАтЕльСтво. Существование $l(p, L, f)$ в $L$ вытекает из теоремы 3.4. Его единственность в случае веса $p$, ограниченного и полунепрерывного сверху, доказывается на основании теоремы 3.6 об альтернансе вполне аналогично тому, как в теореме П. Л. Чебьшева доказывается единственность алгебраического полинома наилучшего приближения для непрерьвности функции $f$ (нужно лишь вместо функции $l(x)-f(x)$, где $l(x)=l((1,1), L, f ; x)$, рассмотреть $(l(p, L, f)-f, p)(x))$. Общий случай сводится к этому заменой веса $p$ его полунепрерывной сверху регуляризацией $\bar{p}$ (см. $(0.0 ; 3))$, поскольку, как уже отмечалось в п. $0.2, E(\bar{p}, L, f)=$ $E(p, L, f), l(\bar{p}, L, f)=l(p, L, f)$.

3.8a. ЗАмЕчАнИЕ. В теореме 3.8 нельзя отказаться от ограниченности веса $p$, на что указывает следующий пример. На $\Delta=[-1,1]$ вес $p$ определим равенством $p(x)=\left(|x|^{-1 / 3},|x|^{-1 / 3}\right)$, в качестве $L$ возьмем семейство всех линейных функций $l(x)=a x+b$ и положим $f(x)=x^{1 / 3}$. Тогда функцией наилучшего приближения для $f$ будет каждая функция вида $l(x)=a x$ при $a \in[0,2]$, причем $E(p, L, f)=1$.

3.8б. ЗАмечание. Теорема 3.8 не может быть обобщена с функционала $|\cdot|_{p, \Delta}$ на произвольные ограниченные сублинейные функционалы $P(\cdot)$, на что указывает пример ограниченного сублинейного функционала $P(f)=\int_{E}|f(x)| d x$ на $C(\Delta)$ при $\Delta=[-1,1], E=[-1,-\varepsilon] \cup[\varepsilon, 1], 0<\varepsilon<1$. Для него и для функции $f \in C(\Delta)$, равной $\operatorname{sign} x$ на $E, L=\{$ const $\}=\mathbb{R}$, алгебраическим полиномом порядка нуль наилучшего приближения для $f$ будет $l(P, L, f) \equiv C$, где $C$-любая постоянная из $[-1,1]$. При этом $E(P ; L, f)=2(1-\varepsilon)>0$.

\section{§3. Вопросы единственности}

Если $E(p, L, f)>0$, где $p$ - ограниченньй знакочувствительньй вес на отрезке $\Delta, L$ - чебьшевское подпространство на $\Delta, f \in C(\Delta)$, то элемент $l(p, L, f)$ существует (см. п. 3.4) и является единственным (см. п. 3.8). Таким образом, при приближении непрерывных функций $f$ на отрезке $\Delta$ элементами чебышевского на $\Delta$ подпространства $L$ вопрос о единственности элемента наилучшего приближения остается открытым лишь в случае $E(p, L, f)=0$.

Следующая теорема решает задачу описания знакочувствительных весов, при равномерных приближениях с которыми непрерывных функций чебышевскими подпространствами элемент наилучшего приближения единствен для каждой функции $f$ независимо от величины $E(p, L, f)$. Стоит обратить внимание на то, что при пользовании ею нужно о чебышевском подпространстве $L$ знать лишь его линейную размерность.

3.9. Теорема. Пусть $L-n$-мерное чебыиевское подпространство на отрезке $\Delta=[a, b],-\infty \leqslant a<b \leqslant+\infty, \quad p=\left(p_{-}, p_{+}\right)$- ограниченный вес на $\Delta$. Тогда для того чтобы каждая функция $f \in C(\Delta)$ имела в $L$ ровно один әлемент наилучшего приближения с весом р, необходимо и достаточно, чтобы множество $\Pi(p)=\operatorname{supp}\left(p_{-}\right) \cap \operatorname{supp}\left(p_{+}\right)$содержало не менее $n$ точек. 
3.9a. ЗАмечаниЕ. Теорема 3.9 в части необходимости верна и без предположения об ограниченности веса $p$ или его конечности (см. доказательство). В части же достаточности это предположение снять нельзя (см. замечание $3.8 \mathrm{a})$.

ДокаЗАТЕЛЬСтво. Необходимость. Допустим противное: есть единственность для каждой $f \in C(\Delta)$, но П $(p)$ состоит не более чем из $n-1$ точек. При $\Pi(p) \neq \varnothing$ пусть $d(x)$ - расстояние от $x$ до $\Pi(p), f(x)=-\omega(L, d(x))$ при $x \in$ $\operatorname{supp}\left(p_{-}\right), f(x)=\omega(L, d(x))$ при $x \in \operatorname{supp}\left(p_{+}\right)$(определение $\omega(L, t)$ см. в п. 2.82)). В случае же $\Pi(p)=\varnothing$ положим $f(x)=-1$ на $\operatorname{supp}\left(p_{-}\right), f(x)=1$ на $\operatorname{supp}\left(p_{+}\right)$. Продолжим функцию $f$ по непрерьвности с $F=\operatorname{supp}(p)=\operatorname{supp}\left(p_{-}\right) \cup \operatorname{supp}\left(p_{+}\right)$ на $\Delta$ так, чтобы она была линейной в дополнительных интервалах к $F$ и при этом постоянной на $[a, \inf F]$, если $a<\inf F$, и на $[\sup F, b]$, если $\sup F<b$. Возьмем какой-нибудь элемент $l \in L, l \not \equiv 0,\|l\|_{\Delta} \leqslant 1$, равный нулю на П $(p)$ (см. п. 2.4). Тогда по определению $\omega(L, t)$ при $-1 \leqslant h=$ const $\leqslant 1$ и $x \in F$ будет

$$
|h l(x)| \leqslant \omega(L, d(x))=|f(x)| .
$$

Поэтому $h l(x)-f(x) \geqslant 0$ на $\operatorname{supp}\left(p_{-}\right), h l(x)-f(x) \leqslant 0$ на $\operatorname{supp}\left(p_{+}\right)$, так что $(h l-f, p)(x) \equiv 0$ на $\Delta$, и $h l(x)$ при любом $h \in[-1,1]$ является элементом наилучшего приближения для $f$ с весом $p$, что невозможно.

Достаточность. Если $E(p, L, f)>0$, то все доказано (см. п. 3.8). Пусть теперь $\Pi(p)$ содержит не менее $n$ точек, а $E(p, L, f)=0$. Тогда (см. п. 2.7а) $W(p, L)<\infty$ и для любой $f \in C(\Delta)$ существует $l(p, L, f)$ (см. п. 3.1а). Ввиду непрерывности $f$ и ее элемента наилучшего приближения все эти элементы на П $(p)$ совпадают с $f$, а значит, и друг с другом. Так как П $(p)$ содержит не менее $n$ точек, то все эти элементы совпадают на $\Delta$. Теорема доказана.

Теорема 3.9 дает необходимое и достаточное условия для единственности элемента наилучшего приближения $l(p, L, f)$ у каждой функции $f \in C(\Delta)$ в случае приближения чебышевским подпространством $L$ с ограниченным знакочувствительным весом $p$ на отрезке $\Delta$. Если число точек $\operatorname{card} \Pi(p)$ множества $\Pi(p)=$ $\operatorname{supp}\left(p_{-}\right) \cap \operatorname{supp}\left(p_{+}\right)$строго меньше $n=\operatorname{dim} L$ и при этом $W(p, L)<\infty$, то в $C(\Delta)$ имеются как функции $f$ с единственным элементом $l(p, L, f)$, так и $f$ с неединственным $l(p, L, f)$ (см. ниже п. 3.16). Теперь уже в критерий элемента наилучшего приближения обязательно должна каким-то образом входить приближаемая функция $f$. Следующая теорема дает такой критерий для крайнего случая $\operatorname{card} \Pi(p)=0$. В случае $0<\operatorname{card} \Pi(p)<n$ эта задача ждет своего решения.

3.10. ТеоремА. Пусть $p=\left(p_{-}, p_{+}\right)$- ограниченный вес на отрезке $\Delta$, $\operatorname{supp}\left(p_{-}\right) u \operatorname{supp}\left(p_{+}\right)$не пересекаются, $L-n$-мерное чебшиевское подпространство на $\Delta, \quad f \in C(\Delta), E(p, L, f)=0$. Тогда для того чтобы әлемент $f \in L$ был единственным в $L$ әлементом наилучшего приближения функиии $f$ с весом $p$, необходимо и достаточно, чтобь на $\Delta$ нашлась возрастающая последовательность из $n+1$ точек, поочередно принадлежащих множествам $\operatorname{supp}\left(p_{-}\right)$и $\operatorname{supp}\left(p_{+}\right)$(начиная с любого из них), в которых $l(x)$ совпадает с $f(x)$. 
ДокаЗАТЕЛЬСтво. Необходимость. Пусть $l=l(p, L, f)$ - единственный в $L$ элемент наилучшего приближения для $f$ с весом $p$. Положим $g_{-}(x)=f(x)$ при $x \in \operatorname{supp}\left(p_{-}\right)$и $g_{-}(x)=-\infty$ при $x \in \Delta \backslash \operatorname{supp}\left(p_{-}\right), g_{+}(x)=f(x)$ при $x \in \operatorname{supp}\left(p_{+}\right)$ и $g_{+}(x)=+\infty$ при $x \in \Delta \backslash \operatorname{supp}\left(p_{+}\right)$. Так как носители $p_{-}$и $p_{+}$не пересекаются, то $g_{-}(x)$ полунепрерывна сверху, а $g_{+}(x)$ полунепрерывна снизу и $g_{-}(x)<g_{+}(x)$ на $\Delta$. Поэтому на $\Delta$ существует такая непрерьвная функция $g(x)$, что $g_{-}(x)<$ $g(x)<g_{+}(x), x \in \Delta$.

Введем вес $q=\left(q_{-}, q_{+}\right)$равенствами

$$
q_{-}(x)=\frac{1}{g(x)-g_{-}(x)}, \quad q_{+}(x)=\frac{1}{g_{+}(x)-g(x)} .
$$

Отметим, что $W(q, L, f)<\infty$. Действительно, по теореме 2.7 , если бы это было не так, то для веса $q$ не выполнялось бы ни условие 2.5, а), ни условие 2.5 б), и так как попарно $p_{-}(x)$ и $q_{-}(x), p_{+}(x)$ и $q_{+}(x)$ обрашаются в нуль одновременно, то и для веса $p$ не выполняюлись бы эти условия, так что и $W(p, L, f)=\infty$ (по той же теореме). Но тогда по теореме 2.1 имеется функция $l_{0} \in L$, для которой $\left\|l_{0}\right\|=1$, $\left|l_{0}\right|_{p, \Delta}=0$, так что при любом $t>0$ имеем

$$
E(p, L, f) \leqslant\left|\left(l+t l_{0}\right)-f\right|_{p, \Delta} \leqslant|l-f|_{p, \Delta}+t\left|l_{0}\right|_{p, \Delta}=E(p, L, f),
$$

т.е., вопреки предположению о единственности $l=l(p, L, f), l+t l_{0}$ - также элемент наилучшего приближения для $f$.

Так как $E(p, L, f)=0$, то $g_{-}(x) \leqslant l(x) \leqslant g_{+}(x)$ всюду на $\Delta$,

$$
g_{-}(x)-g(x) \leqslant l(x)-g(x) \leqslant g_{+}(x)-g(x),
$$

откуда $|l-g|_{q, \Delta} \leqslant 1$. Для элемента $m=l(q, L, g)$ (он сушествует по теореме 3.5, а)) и чисел $Q=E(q, L, g), d_{-}=\min \left\{g(x)-g_{-}(x): x \in \Delta\right\}>0$ и $d_{+}=\min \left\{g_{+}(x)-g(x): x \in \Delta\right\}>0$ получаем неравенства

$$
\begin{gathered}
Q \leqslant 1, \quad-Q \cdot\left(g(x)-g_{-}(x)\right) \leqslant m(x)-g(x) \leqslant Q \cdot\left(g_{+}(x)-g(x)\right), \\
g_{-}(x)+(1-Q)\left(g(x)-g_{-}(x)\right) \leqslant m(x) \leqslant g_{+}(x)-(1-Q)\left(g_{+}(x)-g(x)\right), \\
g_{-}(x)+(1-Q) d_{-} \leqslant m(x) \leqslant g_{+}(x)-(1-Q) d_{+} .
\end{gathered}
$$

Предположим, что $Q<1$. Тогда $g_{-}(x)<m(x)<g_{+}(x)$, и поэтому существует целое семейство линейной размерности $n$ таких элементов $u \in L$, что $g_{-}(x) \leqslant$ $u(x) \leqslant g_{+}(x)$ при всех $x$. Это неравенство равносильно равенству $|u-f|_{p, \Delta}=0$, так что все эти $u$ являются элементами наилучшего приближения для $f$ с весом $p$, что противоречит единственности $l(p, L, f)$.

Итак, $Q=E(q, L, g)=1$. По теореме $3.8 m$ - единственный в $L$ элемент наилучшего приближения для $g$ с весом $q$, т.е. единственньй в $L$ элемент, для которого на $\Delta$ имеем неравенства

$$
g_{-}(x)-g(x) \leqslant m(x)-g(x) \leqslant g_{+}(x)-g(x), \quad g_{-}(x) \leqslant m(x) \leqslant g_{+}(x) .
$$

Поскольку $g_{-}(x) \leqslant l(x) \leqslant g_{+}(x)$, то $m(x) \equiv l(x)$. Функция $l \equiv m$ как элемент наилучшего приближения для $g$ с весом $q$ обладает $(n+1)$-точечным $q$-альтернансом 
по отношению к $g$, т.е. совпадает с $f$ в $n+1$ точках, поочередно принадлежащих $\operatorname{supp}\left(p_{-}\right)$и $\operatorname{supp}\left(p_{+}\right)$.

Достаточность. Если при обозначениях, принятых выше, функция $l(x)$ из $L$, удовлетворяющая на $\Delta$ неравенству $g_{-}(x) \leqslant l(x) \leqslant g_{+}(x)$, совпадает с $f(x)$ в $n+1$ точках, поочередно принадлежаших $\operatorname{supp}\left(p_{-}\right)$и $\operatorname{supp}\left(p_{+}\right)$, то эти точки образуют $q$-альтернанс для $l$ по отношению к $g$. Поэтому $Q=1$ и, следовательно, $l$ - единственный в $L$ элемент наилучшего приближения для $g$ с весом $q$ (см. пп. 3.6 и 3.8 ). Другими словами, $l$ - единственный элемент из $L$, удовлетворяющий на $\Delta$ неравенству $g_{-}(x) \leqslant l(x) \leqslant g_{+}(x)$, эквивалентному равенству $|l-f|_{p, \Delta}=0$. Теорема доказана.

Здесь удобно ввести некоторые определения.

ОПРЕДЕЛЕНИЕ 1. Пусть $A$ и $L-$ два подмножества линейного пространства $\mathscr{L}$, $P$ - сублинейный функционал на $\mathscr{L}$. Скажем, что система (пара) $(P ; L)$ обладает свойством единственности на $A$, если для любого $f \in A$ в $L$ имеется не более одного элемента $l(P ; L, f)$ наилучшего приближения. Если для любого $f \in A$ в $L$ имеется ровно один элемент $l(P ; L, f)$, то скажем, что пара $(P ; L)$ обладает свойством существования и единственности на $A$.

Теперь теорему 3.8 можно переформулировать так:

3.11. ТЕОРемА. Если знакочувствительный вес р на отрезке $\Delta$ ограничен, то сублинейный функиионал $P(\cdot)=|\cdot|_{p, \Delta}(f \in \mathscr{L}=C(\Delta))$ образует с любым чебышевским подпространством $L$ на $\Delta$ пару $(P ; L)$, обладающую свойством существования и единственности на мнохсестве функций $f \in C(\Delta)$, для которых $E(p, L, f)>0$.

ОПРЕДЕЛЕНИЕ 2. Пусть $P$ - сублинейный функционал на линейном нормированном пространстве $\mathscr{L}, L$ и $A$ - множества в $\mathscr{L}$. Назовем пару $(P ; L)$ интерполяuионной на $A$, если для каждого $f \in A$ имеем $E(P ; L, f)=0$ и при этом элемент наилучшего приближения $l(P ; L, f)$ существует и является единственным. Если при этом $P(\cdot)=|\cdot|_{p, E}, \mathscr{L} \subset M(E)$, то будем говорить об интерполящионности пары $(p, L)$.

Легко доказьвается следующее

УТВЕРЖДЕНИЕ. Если $P$ - полунепрерывный снизу сублинейный функционал на линейном нормированном пространстве $\mathscr{L}, L$ - конечномерное подпространство из $\mathscr{L}$, пара $(P ; L)$ интерполяционная, то $W(P ; L)<\infty$.

Действительно, если бы было $W(P ; L)=\infty$, то по теореме 2.1 имелся бы элемент $m \in \mathscr{L}$ с $\|m\|=1$, для которого $P(m)=0$. Если $f \in \mathscr{L}, l=l(P ; L, f), t>0$, TO

$$
E(P ; L, f) \leqslant P(l+t m-f) \leqslant P(l-f)+t P(f)=E(P ; L, f),
$$

так что $l+t m$ - также элемент наилучшего приближения для $f$.

Приведем примеры интерполящионных пар.

Пример 1. $L-n$-мерное чебьшевское подпространство на отрезке $\Delta$, а $P(f)=$ $|f|_{p, \Delta}$, где вес $p=\left(p_{-}, p_{+}\right)$является невырожденным в $n$ точках отрезках $\Delta$ и вырожденным в остальных его точках. Для любой функции $f \in A=\mathscr{L}=M(\Delta)$ 
в этом случае $l(P ; L, f ; x)$ - интерполящионный полином по системе $\left\{\varphi_{k}\right\}$, порождающей $L$, с узлами интерполяции в упомянутых $n$ точках.

Пример 2. $L$-трехмерное чебышевское подпространство квадратных трехчленов $l(x)=a x^{2}+b x+c$ на отрезке $\Delta=[0,1], A=\mathscr{L}=C(\Delta)$,

$$
P(f)=|f(0)|+|f(1)|+\left|\int_{\Delta} f(x) d x\right| .
$$

При $t \in(-\infty,+\infty)$ полином

$$
l(x)=f(0)(1-x)+f(1) x+t x(1-x)
$$

совпадает с $f$ на концах $\Delta$, и каждый квадратный трехчлен, совпадающий с $f$ на конщах $\Delta$, имеет такой вид. Поскольку при $x \in(0,1)$ с ростом $t$ от $-\infty$ до $+\infty$ $l(x)$ возрастает от $-\infty$ до $+\infty$, то ровно при одном значении $t$ интеграл от $l-f$ по отрезку $\Delta$ обрашается в нуль. Поэтому $E(P ; L, f)=0$, и полином $l(P ; L, f)$ единствен.

В случае чебышевского подпространства $L$ знакочувствительные веса, образующие интерполящионные пары с $L$, имеют простое описание.

3.12. ТЕОРемА. Пусть $L-n$-мерное чебишевское подпространство на отрезке $\Delta, \quad p=\left(p_{-}, p_{+}\right)$- вес на $\Delta$ (не обязательно конечный). Тогда для того чтобы пара $(p, L)$ была интерполячионной на $C(\Delta)$, необходимо и достаточно, чтобы вес $р$ был невырожденным в некоторых $n$ точках отрезка $\Delta$ и вырожденным в остальных его точках.

ДоказАТЕЛЬСтво. Необходимость. Пусть для любой функции $f \in C(\Delta)$ имеем $E(p, L, f)=0$, и элемент $l(p, L, f)$ единствен. Тогда по 3.9а $\Pi(p)($ см. $(3.5 ; 0))$ содержит не менее $n$ точек, ровно $n$ из которых мы фиксируем и обозначим $\left\{x_{k}\right\}_{1}^{n}$. Из 2.7а имеем $W(p, L)<\infty$. Далее, ввиду непрерывности функций $f$ и $l:=$ $l(p, L, f)$ на $\Delta$ имеем $l\left(x_{k}\right)=f\left(x_{k}\right), k=1, \ldots, n$. Так как подпространство $L$ $n$-мерное чебышевское, то последними равенствами $l(x)$ определена однозначно и не зависит от значений $f$ в остальных точках $\Delta$.

Предположим, что не выполняется условие необходимости теоремы, т.е. (см. п. 2.5) на $\Delta$ имеется $n+1$ точка, где положительны попеременно $p_{-}$и $p_{+}$. Обозначим через $t$ какую-нибудь из этих точек, отличную от точек $x_{k}$, и пусть, например, $p_{+}(t)>0$. Изменим в случае необходимости функцию $f$ так, чтобы было $l(t)>f(t)$. Для такой функции $f$, очевидно, $E(p, L, f)>0$, и мы пришли к противоречию с условием теоремы.

Достаточность следует из сушествования и единственности интерполящионного полинома по чебышевской системе (см., например, [14]).

\section{§4. Множества элементов наилучшего приближения}

Здесь мы займемся геометрическими свойствами множества $A(P ; L, f)$ (и $\left.A(p, L, f):=A\left(|\cdot|_{p, E} ; L, f\right)\right)$ всех элементов наилучшего приближения элемента $f \in \mathscr{L}$ элементами из $L \subset \mathscr{L}$ относительно функционала $P$. Отметим следующее: 
а) если множество $L$ выпукло в $\mathscr{L}$, то $A(P ; L, f)$ также выпукло;

б) если $L$ замкнуто, а функционал $P$ полунепрерывен снизу на $\mathscr{L}$, то и $A(P ; L, f)$ также замкнуто.

Действительно, если $L$ выпукло, $l, m \in A(P ; L, f), t \in[0,1]$, то

$E(P ; L, f) \leqslant P((t l+(1-t) m)-f) \leqslant t P(l-f)+(1-t) P(m-f)=E(P ; L, f)$, откуда $t l+(1-t) m \in A(P ; L, f)$, так что $A(P ; L, f)$ выпукло. Замкнутость же $A(P ; L, f)$ при замкнутом $L$ непосредственно следует из определения полунепрерывности снизу функционала $P$.

ЗАМЕЧАнИЕ. Если $L$ - некоторое подмножество линейного нормированного пространства $\mathscr{L}, P$ - конечный сублинейный функционал на $\mathscr{L}$, то из конечности $W(P ; L)$ следует конечность диаметра $\operatorname{diam} A(P ; L, f)$ множества $A(P ; L, f)$ для любого $f \in \mathscr{L}$ с непустым $A(P ; L, f)$.

Действительно, если $\operatorname{diam} A(P ; L, f)=\infty$ и $l_{n} \in A(P ; L, f),\left\|l_{n}\right\| \rightarrow \infty$, то

$$
\frac{P\left(l_{n}\right)}{\left\|l_{n}\right\|} \leqslant \frac{P\left(l_{n}-f\right)+P(f)}{\left\|l_{n}\right\|}=\frac{E(P ; L, f)+P(f)}{\left\|l_{n}\right\|} \rightarrow 0,
$$

так что $S(P ; L)=0$ и $W(P ; L)=\infty$.

3.13. ТеОРемА. Пусть $L$ - конечномерное линейное подпространство некоторого линейного нормированного пространства $\mathscr{L}, P$ - полунепрерывный снизу сублинейный функционал на $\mathscr{L}$. Тогда равенство $W(P ; L)=\infty$ необходимо и достаточно для неединственности әлемента наилучшего приближения $l(P ; L, f)$ у каждого әлемента $f \in \mathscr{L}$ с непустым $A(P ; L, f)$. Это равенство необходимо и достаточно также для бесконечности $\operatorname{diam} A(P ; L, f)$ у каждого такого $f$.

ДоказАтельство. Необходимость. Так как $l=0$ - элемент наилучшего приближения для $f=0$ и $l(P ; L, 0)$ не единственный, то в $L$ имеется $l \neq 0$, для которого $P(l)=0$, откуда $W(P ; L)=\infty$ (см. п. 2.1).

Достаточность. Если $W(P ; L)=\infty$, то (по 2.1) имеется $l_{0} \in L, l_{0} \neq 0$, для которого $P\left(l_{0}\right)=0$. Поэтому если $l=l(P ; L, f)$, то $l+t l_{0}$ при любом $t>0$-также элемент наилучшего приближения для $f$, так как

$$
E(P ; L, f) \leqslant P\left(\left(l+t l_{0}\right)-f\right) \leqslant P(l-f)+t P\left(l_{0}\right)=E(P ; L, f) .
$$

Поскольку $\left\|l+t l_{0}\right\|_{E} \rightarrow \infty$ при $t \rightarrow \infty$, то $\operatorname{diam} A(P ; L, f)=\infty$.

Следующие теоремы дают оценку диаметра множества $A(P ; L, f)$ в случае $W(P ; L)<\infty$.

3.14. ТЕОРемА. Пусть $L$ - выпуклое подмножество некоторого линейного нормированного пространства $\mathscr{L}, P$ - сублинейный функционал на $\mathscr{L}$, $W(P ; L)<\infty, \quad f \in \mathscr{L}$. Тогда

$$
\operatorname{diam} A(P ; L, f) \leqslant 2 W(P ; L)(E(P ; L, f)+E(P ; L,-f)),
$$

и более общо: если $g_{k}, h_{k} \in L, \quad P\left(g_{k}-f\right) \rightarrow E(P ; L, f), \quad P\left(h_{k}-(-f)\right) \rightarrow$ $E(P ; L,-f) \quad(f \in \mathscr{L}, k \rightarrow \infty)$, mo

$$
\varlimsup_{k \rightarrow \infty}\left\|g_{k}+h_{k}\right\| \leqslant W(P ; L)(E(P ; L, f)+E(P ; L,-f)) .
$$


ДокаЗАТЕЛЬСтво. Если $W(P ; L)<\infty, f \in \mathscr{L}, r>0$ и $g, h \in L$ таковы, что $P(g-f)<E(P ; L, f)+r, P(h-(-f))<E(P ; L,-f)+r$, то $(g+h) / 2 \in L$,

$$
\begin{aligned}
\|g+h\| & =2\left\|\frac{g+h}{2}\right\| \leqslant 2 W(P ; L) \cdot P\left(\frac{g+h}{2}\right) \\
& \leqslant W(P ; L)(P(g-f)+P(h-(-f))) \\
& <W(P ; L)(E(P ; L, f)+E(P ; L,-f)+2 r) .
\end{aligned}
$$

Отсюда следует $(3.14 ; 2)$. При $l_{1}, l_{2} \in A(P ; L, f)$ и $g_{k}=l_{1}$ или $g_{k}=l_{2}$ из $(3.14 ; 2)$ получаем $(3.14 ; 1)$

$$
\left\|l_{1}-l_{2}\right\| \leqslant \varlimsup\left(\left\|l_{1}+h_{k}\right\|+\left\|l_{2}+h_{k}\right\|\right) \leqslant 2 W(P ; L)(E(P ; L, f)+E(P ; L,-f)) .
$$

Из теорем 3.11 и 3.14 получаем

3.15. СЛЕДСТВИЕ. Пусть $L$ - чебышевское подпространство на отрезке $\Delta, p$ - ограниченный знакочувствительный вес на $\Delta, W(p, L)<\infty$. Тогда для любой функции $f \in C(\Delta)$ имеем неравенство

$$
\operatorname{diam} A(p, L, f) \leqslant 2 W(p, L) E(p, L,-f) .
$$

(Это неравенство тривиально при $E(p, L, f)>0$, а при $E(p, L, f)=0$ оно следует из $(3.14 ; 1)$.)

Отсюда получаем

3.16. СлЕДСТвИЕ. Если при условиях утверждения 3.15 у некоторой $f \in$ $C(\Delta)$ функция наилучшего приближения не единственна, то $y-f$ ее функция наилучшего приближения единственна и при этом $E(p, L,-f)>0$ (cм. n. 3.8).

3.17. СлЕДСТВИЕ. Если при условиях теоремы 3.14 для некоторого $f \in \mathscr{L}$ имеем $E(P ; L, f)=E(P ; L,-f)=0$, то и у $f$, и $y-f$ соответствующий әлемент наилучшего приближения, если он существует, является единственныл, причем если существует $l(P ; L, f)$, то существует и $l(P ; \bar{L},-f)=$ $-l(P ; L, f)(\bar{L}-$ замыкание в $\mathscr{L}$ множества $L)$.

Это прямо вытекает из $(3.14 ; 2)$. В частности (при $\left.P(f)=|f|_{p, E}\right)$, если $p=$ $\left(p_{-}, p_{+}\right)$- знакочувствительный вес на $E \subset \mathbb{R}, p^{*}=\left(p_{+}, p_{-}\right), L$ - конечномерное подпространство из $C(E), W(p, L)<\infty$ и $E(p, L, f)=E\left(p^{*}, L, f\right)=0$ для некоторой функции $f \in C(E)$, то $l(p, L, f)$ и $l\left(p^{*}, L, f\right)$ определены единственньм образом и совпадают (заметим, что $\left.\left|\left(l-f, p^{*}\right)(x)\right|=|(-l-(-f), p)(x)|\right)$.

3.18. Теорема 3.14 и ее следствия оправдывают следующее

ОПРЕДЕЛЕнИЕ. Пусть $P$ - неотрицательный функционал в линейном пространстве $\mathscr{L}$, множество $A$ центрально симметрично (т.е. вместе с каждым элементом $f$ оно содержит и элемент $-f), L \subset \mathscr{L}$. Если при любом $f \in A$ либо $f$, либо хотя бы $-f$ имеет в $L$ не более одного элемента наилучшего приближения, то скажем, что система (пара) $(P ; L)$ (соответственно $(p, L)$ при $\left.P(\cdot)=|\cdot|_{p, E}\right)$ обладает свойством альтернативной единственности на $A$. 
3.19. ТеоремА. Если $L$ - чебышевское подпространство на отрезке $\Delta$, $p$ - ограниченный знакочувствительный вес на $\Delta$, то условие $W(p, L)<\infty$ необходимо и достаточно для альтернативной единственности системы $(p, L)$ на $C(\Delta)$.

ДокАЗАТЕЛЬСтво. Если имеется альтернативная единственность, то $W(p, L)<\infty$ (см. п. 3.13). Достаточность следует из п. 3.16.

Следуюшая теорема выявляет зависимость диаметра $A(p, L, f)$ от замкнутых носителей $\operatorname{supp}\left(p_{-}\right)$и $\operatorname{supp}\left(p_{+}\right)$компонент веса $p$.

Введем обозначения. Пусть $A \subset E \subset \mathbb{R}$ ( $A$ может быть и пустым) и на $E$ определены функции $g(x) \leqslant G(x), \varphi(x)$ и функции некоторого семейства $L$. Через $L(A, \varphi)$ обозначим множество всех $l \in L$, совпадающих с $\varphi$ на $A$ (если $A$ пусто, то $L(A, \varphi):=L)$. Через $B(g, G ; L)$ обозначим множество всех таких $l \in L$, что $g(x) \leqslant l(x) \leqslant G(x)$ при всех $x \in E$. Положим

$$
b(g, G ; L):=\sup \{|l(x)-m(x)|: l, m \in B(g, G ; L), x \in E\} .
$$

3.20. Теорема. Пусть $L$ - линейное подпространство из $C(\Delta)(\Delta=$ $[a, b]), p$-знакочувствительный вес на $\Delta, k_{-}(x)$ и $k_{+}(x)$ - характеристические функиии соответственно множсеств $\operatorname{supp}\left(p_{-}\right)$и $\operatorname{supp}\left(p_{+}\right), k=\left(k_{-}, k_{+}\right)$, $f \in C(\Delta)$. Тогда если $E(p, L, f)=0$ и $A(p, L, f) \neq \varnothing$, mо

$$
\operatorname{diam} A(p, L, f) \leqslant \inf \left\{b\left(-\frac{1}{k_{-}}, \frac{1}{k_{+}} ; L(A, 0)\right) \cdot E(k, L(A,-f),-f): A \subset \Pi(p)\right\} ;
$$

в частности,

$$
\operatorname{diam} A(p, L, f) \leqslant b\left(-\frac{1}{k_{-}}, \frac{1}{k_{+}} ; L\right) \cdot E(k, L,-f) \leqslant 2 W(k, L) \cdot E(k, L,-f) .
$$

ДоКАЗАТЕЛЬСТВО. Положим $f_{-}(x)=f(x)$ при $x \in \operatorname{supp}\left(p_{-}\right)$и $f_{-}(x)=-\infty$ при остальных $x, f_{+}(x)=f(x)$ при $x \in \operatorname{supp}\left(p_{+}\right)$и $f_{+}(x)=+\infty$ при остальных $x$. Так как $E(p, L, f)=0$, то $l \in A(p, L, f)$ тогда и только тогда, когда $f_{-}(x) \leqslant l(x) \leqslant f_{+}(x)$ при всех $x \in \Delta$; в частности, $l(x)=f(x)$ при $x \in \Pi(p)$ (обратим внимание на то, что эти соотношения являются ограничениями лишь при $f_{-}(x)>-\infty$ или $f_{+}(x)<+\infty$ и что они учитывают лишь множества $\operatorname{supp}\left(p_{-}\right)$и $\operatorname{supp}\left(p_{+}\right)$, но не значения $p_{-}(x)$ или $p_{+}(x)$ на них). Поэтому при $A \subset \Pi(p)$ и $m \in L$ имеем равенства

$$
\begin{aligned}
\operatorname{diam} A(p, L, f) & =b\left(f_{-}, f_{+} ; L\right)=b\left(f_{-}, f_{+} ; L(A, f)\right) \\
& =b\left(f_{-}+m, f_{+}+m ; L(A, f+m)\right) .
\end{aligned}
$$

Заметим, что из непустоты $L(A, f)$ вытекает непустота $L(A,-f) \subset L$. Пусть $Q:=$ $E(k, L(A,-f),-f), \varepsilon>0$, a $m \in L(A,-f)$ таково, что

$$
|m+f|_{k, \Delta}=|m-(-f)|_{k, \Delta} \leqslant Q+\varepsilon .
$$


Из $(3.20 ; 3)$ получаем соотношения

$$
\begin{aligned}
\operatorname{diam} A(p, L, f) & =b\left(f_{-}+m, f_{+}+m ; L(A, 0)\right) \leqslant b\left(-\frac{Q+\varepsilon}{k_{-}}, \frac{Q+\varepsilon}{k_{+}} ; L(A, 0)\right) \\
& =(Q+\varepsilon) b\left(-\frac{1}{k_{-}}, \frac{1}{k_{+}} ; L(A, 0)\right)
\end{aligned}
$$

откуда следует $(3.20 ; 1)$. Первое из неравенств $(3.20 ; 2)$ получается из $(3.20 ; 1)$ при пустом $A$, второе - из первого и из определений $|\cdot|_{k, \Delta}$ и $W(k, L)$.

В заключение отметим, что имеются исследования по некоторым вопросам $и$ - $^{-}$ тегральных приближений с постоянным знакочувствительным весом (см. [22]) и приближений относительно обобшенного знакочувствительного веса [23].

Результаты, касающиеся устойчивости наименьших уклонений и наилучших приближений непрерывных функций при вариациях знакочувствительного веса, а также приложения теории знакочувствительных аппроксимаций к некоторьм экстремальным задачам, будут опубликованы во второй части работы.

\section{Список литературы}

1. Долженко Е.П., Севастьянов Е.А. Аппроксимация со знакочувствительным весом // Российская конф. по нелинейному анализу. Махачкала, 1992. Махачкала: ДГУ, 1994. C. $42-43$.

2. Долженко Е. П. Знакочувствительные аппроксимации чебышевскими подпространствами // Тез. докл. школы "Теория функций. Дифференциальные уравнения в математическом моделировании". Воронеж, 25 января - 3 февраля 1993 г.. Воронеж: ВГУ, 1993. С. 50 .

3. Долженко Е. П., Севастьянов Е. А. Знакочувствительные аппроксимации (пространство знакочувствительных весов, жесткость и свобода системы) // Докл. АН. 1993. Т. 332. №6. C. $686-689$.

4. Долженко Е.П., Севастьянов Е. А. Знакочувствительные аппроксимации (вопросы единственности и устойчивости) // Докл. АН. 1993. Т. 333. № 1. С. 5-7.

5. Долженко E. П., Севастьянов E. А. Метрические пространства полунепрерывных функций // Матем. заметки. 1994. Т. 55. № 3. С. 48-58.

6. Долженко Е. П., Севастьянов Е. А. Об определении чебышевских ужей // Вестн. МГУ. Матем. Мех. 1994. № 3. С. 49-59.

7. Долженко Е. П. Знакочувствительные аппроксимации // Тез. докл. "Конструктивная теория функций и ее приложения". Махачкала, 1994. Махачкала: ДГУ, 1994. С. 38-40.

8. Долженко Е. П. Знакочувствительные аппроксимации // Тез. докл. Воронежская зимняя математическая школа - 1995. "Современные методы теории функций и смежные проблемы прикладной математики и механики". Воронеж: ВГУ, 1995. С. 89.

9. Долженко Е. П. Знакочувствительные аппроксимации // Тез. докл. Международная конференция "Функциональные пространства, теория приближений, нелинейный анализ”, посв. 90-летию акад. С. М. Никольского. М.: ПАИМС, 1995. С. 114-116.

10. Севастьянов E. A. О существовании и устойчивости чебышевских ужей // Тез. докл. "Конструктивная теория функций и ее приложения". Махачкала, 1994. Махачкала: ДГУ, 1994. С. 102-104.

11. Minkowski H. Theorie der konvexen Körper, insbesondere Begrundung ihres Oberflachenbegriffs // Ges. Abh. 1911. B.2. P. 131-229.

12. Крейн М. Г., Нудельман А. А. Проблема моментов Маркова и экстремальные задачи. М.: Наука, 1973.

13. Сендов Б. Хаусдорфовы приближения. София: Изд-во Болгарской АН, 1979. 
14. Дзядык В. К. Введение в теорию равномерного приближения функций полиномами. М.: Наука, 1977.

15. Севастьянов E. A. Теорема Хаара и ее обобщение для знакочувствительных аппроксимаций // Тез. докл. Международная конференция "Функциональные пространства, теория приближений, нелинейный анализ", посв. 90-летию акад. С. М. Никольского. М.: ПАИМС, 1995. С. 244-245.

16. Севастьянов $E$. A. Проблема единственности элементов наилучшего приближения для знкочувствительных аппроксимаций // Тез. докл. "Теория функций и ее приложения" (школа-конференция, 15-22 июня 1995 г., Казань). Казань: Изд-во Казанского фонда "Математика", 1995. С. 57-60.

17. Севастьянов E. А. О проблеме Хаара для знакочувствительных аппроксимаций // Матем. сб. 1997. Т. 188. № 2. С. 95-128.

18. Бернштейн C.H. Экстремальные свойства полиномов и наилучшее приближение непрерывных функций одной вещественной переменной. М.-Л.: ОНТИ, 1937.

19. Чебышев П. Л. Вопросы о наименьших величинах, связанные с приближенным представлением функций. Полное собрание сочинений. Т. 2. М.-Л.: Изд-во АН СССР, 1948.

20. Vallée-Poussin Ch.-J. Lecon sur l'approximation des fonctions d'une variable réelle. Paris: Gauthier-Villars, 1919.

21. Куратовский К. Топология. Т. 1. М.: Мир, 1966.

22. Бабенко В. $\Phi$. Несимметричные приближения в пространствах суммируемых функций // Укр. матем. журн. 1982. Т. 34. № 4. С. 409-419.

23. Симонова И. Э., Симонов Б. В. О полиноме наилучшего несимметричного приближения в пространстве Орлича // Изв. Вузов. Сер. матем. 1993. № 11 (378). С. 50-56.

Поступило в редакцию 27.V.1997 